\title{
In Silico Modelling: Electronic Properties of Phosphorene Monoflakes and Biflakes Substituted With Al, Si, and S Heteroatoms
}

\section{Cesar Gabriel Vera de la Garza}

UNAM IIM: Universidad Nacional Autonoma de Mexico Instituto de Investigaciones en Materiales

\section{Luis Daniel Solis Rodriguez}

UNAM IIM: Universidad Nacional Autonoma de Mexico Instituto de Investigaciones en Materiales

\section{Serguei Fomine}

UNAM IIM: Universidad Nacional Autonoma de Mexico Instituto de Investigaciones en Materiales

Wilmer Esteban Vallejo Narváez ( $\nabla$ wilmervall@gmail.com )

UNAM IIM: Universidad Nacional Autonoma de Mexico Instituto de Investigaciones en Materiales https://orcid.org/0000-0002-3712-0618

\section{Research Article}

Keywords: Doped phosphorene nanoflakes, DFT, silicon, aluminium, sulfur

Posted Date: February 23rd, 2021

DOI: https://doi.org/10.21203/rs.3.rs-215500/v1

License: (9) This work is licensed under a Creative Commons Attribution 4.0 International License. Read Full License 


\title{
In silico modelling: electronic properties of phosphorene monoflakes and biflakes substituted with $\mathrm{Al}, \mathrm{Si}$, and $\mathrm{S}$ heteroatoms
}

\author{
Cesar Gabriel Vera de la Garza, ${ }^{a}$ Luis Daniel Solis Rodriguez, a Serguei Fomine ${ }^{\text {a }}$ and Wilmer E. \\ Vallejo Narváez *a \\ a Instituto de Investigaciones en Materiales, Universidad Nacional Autónoma de México, \\ Apartado Postal 70-360, CU, Coyoacán, México DF 04510, México.
}

*E-mail: wilmervall@gmail.com

\begin{abstract}
This contribution explores the systematic substitution of monoflakes (Mfs) and biflakes (Bfs) phosphorene with aluminum, silicon, and sulfur. All this was investigated using functional TPSS and CASSCF calculations. Al and Si substitution produces significant structural changes in both Mfs and Bfs compared to S-substituted and pristine systems. However, in Mfs, all heteroatoms generate a decrease in band gap and the ionization potentials (IP), and an increase in electron affinity (EA) in comparison with pristine phosphorene. Al doping improves the hole mobility in the phosphorene monoflake, while Si and S substitutions exhibit a similar behavior on EAs and reorganization energies. For Bfs, the interlaminar interactions Si-Si and Al-P cause structural changes and higher binding energies for Si-Bfs and Al-Bfs. Regarding the electronic properties of Bfs, substitution with Si does not produce significant variations in the band gap. However, it conduces to the formation of hole transport materials concerning its monolayer counterpart. It also is observed in Al-systems, whereas for S-complexes, no correlation was identified between the doping level and reorganization energies. Also, the substitution with $\mathrm{Al}$ and $\mathrm{S}$ leads to an opposite behavior of the band gap and IP values, while the variation in EA is similar. In summary, the nature of heteroatom and the doping degree can modify the semiconductor character and electronic properties of phosphorene mono- and the biflakes, whose trends are closely related to the atomic properties of heteroatoms considered. Overall, these computational calculations provide significant insights into the study of doped phosphorene materials.
\end{abstract}

Keywords: Doped phosphorene nanoflakes, DFT, silicon, aluminium, sulfur.

\section{Introduction}

Graphene synthesized by graphite monolayers separating ${ }^{1-3}$ has become a remarkable material. Since its preparation, several studies have been conducted attempting to understand their electronic, thermal, and mechanic properties. ${ }^{24-9}$ Among them, one of the most important is its high mobility of charge carriers, ${ }^{10}$ which makes graphene an excellent material for optoelectronic devices. Nevertheless, graphene has no band 
gap due to its semi-metallic nature. A non-zero band gap is needed to control charge carriers with an external electrical field. A similar system to graphite/graphene is black phosphorous which is one of its allotropes and is composed of layers bonded by intermolecular forces. ${ }^{11}$ It is known that black phosphorous is a semiconductor with a non-zero energy band gap (approximately $2 \mathrm{eV}$ ) according to the literature. ${ }^{12}$

More than six years ago phosphorous monolayers were successfully isolated, and that material was called "phosphorene". ${ }^{11,12}$ According to the literature, ${ }^{13}$ this species has an energy band gap that is between 0.6 and $1.5 \mathrm{eV}$. It is also known that small changes in black phosphorous structure lead to large variations in the electronic properties of phosphorous and phosphorene. This new material denominated as blue phosphorene was predicted in 2014 and has a band gap of $2 \mathrm{eV}$ suitable for a wide range of applications in optoelectronics. ${ }^{14}$ One of the several attractive properties of phosphorene and blue phosphorene is the strong sensibility of the band gap to the physical/mechanical deformation of the material. The electronic behavior of phosphorene can be switched from semiconductor to metal only by mechanical deformation. ${ }^{15,16}$

Various phosphorene allotropies almost as stables as both mentioned before having been predicted, but only black and blue phosphorenes have been obtained experimentally. All these diverse allotropes have different electronic properties. ${ }^{17-19}$ Nevertheless, very few theoretical studies have been conducted, and in most of them, the periodic boundary condition model has been used for one- and two-dimensional systems. ${ }^{20}$ A few studies include pristine or substituted phosphorene nanoflakes (NFs). ${ }^{21}$ These systems are potentially suitable for optoelectronics not just because the NFs can be of different sizes and shapes compared with one- and two-dimensional periodical systems, but because the analysis of NFs allows for calculation of charged species and their corresponding properties. Furthermore, NFs also permits studying the dynamic behavior of molecules on surfaces. ${ }^{21}$

We consider a previous study ${ }^{22}$ as a starting point, which focused on doped systems but only in monoflakes phosphorene with $\mathrm{Al}$ and $\mathrm{Si}$. The authors found out that the doping of phosphorene nanoflakes with $\mathrm{Al}$ and Si changes its geometry. They also observed that edge doping complexes are less stable than those with inside doping for both $\mathrm{Al}$ and Si. Concerning the electronic properties, the doping impacts only moderately Ionization Potentials (IPs) while Electron Affinities (EAs) are changed much more. Based on above, the present work centred on the theoretical study of the electronic structure of pristine and substituted phosphorene nanoflakes with elements such as $\mathrm{Al}, \mathrm{Si}$, and $\mathrm{S}$. All this to analyze the effect of elements with a higher or lesser electronegativity and size concerning the $\mathrm{P}$ atom. And consequently, understand how the substitution with heteroatoms modifies the electronic properties such as IP, EA, band gap, NBO charges and reorganization energies. Additionally, our study contemplates analysing of doped phosphorene biflakes. Also, we expect these arrangements to be more easily experimentally obtained compared to monoflakes. Thus, it is appropriate to model and understand their properties.

For the geometry optimization, we used the Density Functional Theory (DFT), while Complete Active Space (CASSCF) was utilized only to determine the nature of the ground state for the systems with unstable closed-shell singlet ground states. The combination of these methodologies allows modelling relatively large systems at an acceptable computational cost. 


\section{Computational details}

Based on previous studies, 20,22 the size selected for the monoflakes was 192 atoms, and the double for biflakes. Conformational analysis of biflakes was carried on because there are at least four ways in which the monoflakes could stack to each other. The lowest energy conformation was found to be zigzag stacking (P-Bf shown in Fig. 3).

TPSS is a non-empirical meta functional specially gives a good description of solids and surfaces. ${ }^{23,24}$ It can correctly predict the bond lengths, valence angles, electron affinities, ionization potentials, and other parameters of solids. The geometry optimizations and properties calculations were performed using D3BJ dispersion-corrected TPSS in combination with def2-SVP basis set in Turbomole v7.325 and Gaussian 16 revision A $3^{26}$ codes. In previous studies, ${ }^{27}$ it was demonstrated that the basis set def2-SVP yields good results for this kind of system without compromising the computational cost. The frequency calculates were run for all studied systems to ensure that a minimum (no imaginary frequencies) have been located. The Total density of states (TD-DOS) for Mfs and Bfs were all calculated by Multiwfn 3.8.28

Mononanoflake systems with 4, 6, 8, 10 and 12 substitutions were built for each atom (Al, Si and S). The substitution pattern was made seeking to preserve the symmetry of the nanoflakes. For biflake scaffolds, the previously described monoflakes were used.

For each species, singlet and triplet states were optimized to determine the corresponding ground-state. The stability calculations for all closed-shell singlets were performed and, if it was unstable, the structure was reoptimized as an Open-Shell Singlet (OSS) using the broken symmetry approach. To evaluate the multiconfigurational character of the studied systems, RASSCF single-point energy calculations were carried out for the systems that showed triplet instability of the closed-shell singlet state. ${ }^{29}$ The active space consisting of 20 electrons and 20 orbitals there was applied. These calculations were carried out with Gaussian 16 rev. A3. ${ }^{26}$ The RAS model employed was RAS1 and RAS3 spaces, which consisted of 6 allowing up to 2 holes in RAS1 and up to 2 electrons in RAS3. The rest of the orbitals w as in RAS2 space. 6-31G*(5d) basis set was used for calculations.

Finally, it is known that in the solid-state, the hole mobility is related to the internal reorganization energy $(\lambda)$, which also is a factor involved in the conductivity of the nanoflakes. ${ }^{30}$ We determined the hole reorganization energy $\left(\lambda_{+}\right)$and electron reorganization energy $\left(\lambda_{-}\right)$, for phosphorene mono- and biflakes, whose expression are:

$$
\lambda_{+}=\left(E_{n}{ }^{+}-E_{n}\right)+\left(E_{+}^{n}-E_{+}\right)
$$

where $E_{n}$ and $E_{+}$correspond to the energies of the neutral and cationic species in their equilibrium geometries, respectively, while $\mathrm{E}_{\mathrm{n}}{ }^{+}$and $\mathrm{E}_{+}{ }^{\mathrm{n}}$ represent the energies of the neutral and cationic species with the geometries of the cation and neutral compounds, respectively. For electron reorganization energy, it can be defined as:

$$
\lambda_{-}=\left(E_{n}^{-}-E_{n}\right)+\left(E_{-}^{n}-E_{-}\right)
$$


here, $E_{n}$ and $E_{-}$are the energies of the neutral and anion species in their lowest energy geometries, respectively, while $E_{n}{ }^{-}$and $E_{-}{ }^{n}$ are the energies of the neutral and anion species with the geometries of the cation and neutral species, respectively.

\section{Results and discussion}

\section{Geometries of neutral monoflakes and biflakes}

Fig. 1 shows optimized structures for the unsubstituted system, P-Mf, and those substituted with 8 atoms of $\mathrm{Al}, \mathrm{Si}$, and S. Other monoflakes, Mfs, are shown in Fig. S1 in the ESI. The dopant position (inside) and its distribution is based on previous work ${ }^{21}$ in order to avoid the formation of Al-Al, Si-Si and S-S intramolecular bonds. In the three cases the substitution is the same, as indicated in Fig. 1, so that the results can be comparable. According to the stability calculations, the ground states of almost all systems are closedshell singlet. Only P(4-S), P(4-Si) and $\mathbf{P ( 1 2 - S i ) ~ s h o w e d ~ t r i p l e t ~ i n s t a b i l i t y ~ o f ~ t h e ~ w a v e ~ f u n c t i o n , ~ t h e r e f o r e , ~ t h e y ~}$ were computed as OSS species. In this case, RASSCF calculations indicate that the singlet state is more stable than the triplet.

Considering a structural comparison of the systems (Fig. 1), P(8-S), $\mathbf{P ( 8 - S i ) ~ a n d ~} \mathbf{P ( 8 - A l )}$ are different from each other, whose order of planarity is $\mathbf{P}-\mathbf{M f}>\mathbf{P}(\mathbf{8}-\mathbf{S})>\mathbf{P}(\mathbf{8}-\mathbf{S i})>\mathbf{P}(\mathbf{8}-\mathbf{A l})$. This trend is preserved for the other degrees of substitution, 4, 6, 10, and 12 as shown in Fig 2. Along with the results, the doping with the sulfur atom leads to spatially similar nanoflakes (NFs) to the unsubstituted one as shown in Fig. 1 and 2. Hence, the substitution with $\mathrm{Al}$ and $\mathrm{Si}$ atoms causes significant structural modifications, while $\mathrm{S}$ atoms do not generate a relevant change. This trend agrees with the radius of the heteroatoms, whose ascending order is $\mathrm{S}>\mathrm{Si}>\mathrm{Al}$. The similar values of the deviation from plane of the unsubstituted system and the Sulfursubstituted monoflakes, S-Mf, can be explained by the comparable radius of the $\mathrm{P}$ and $\mathrm{S}$ atoms. The large structural change in Al-substituted systems, Al-Mfs, may be due to the polar covalent character of the Al-P bonds, caused by the difference in electronegativities between $\mathrm{Al}$ and $\mathrm{P}$.

(a)

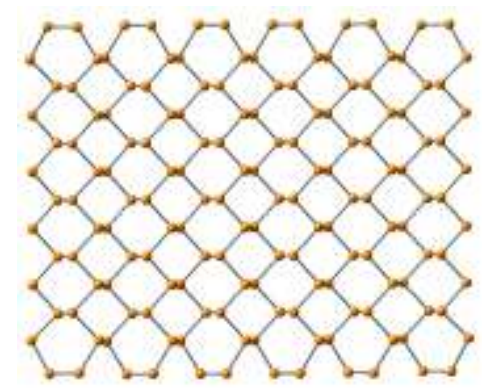

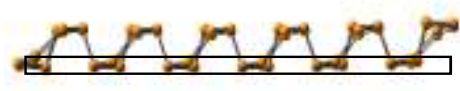

$1.3 \AA$ 
(b)

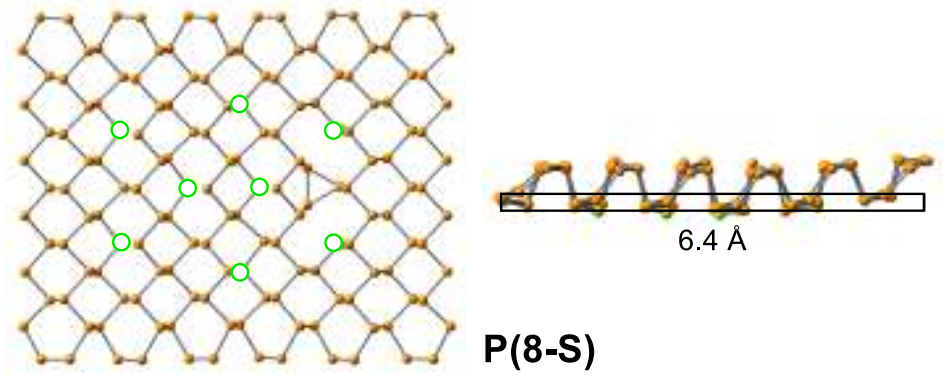

(c)

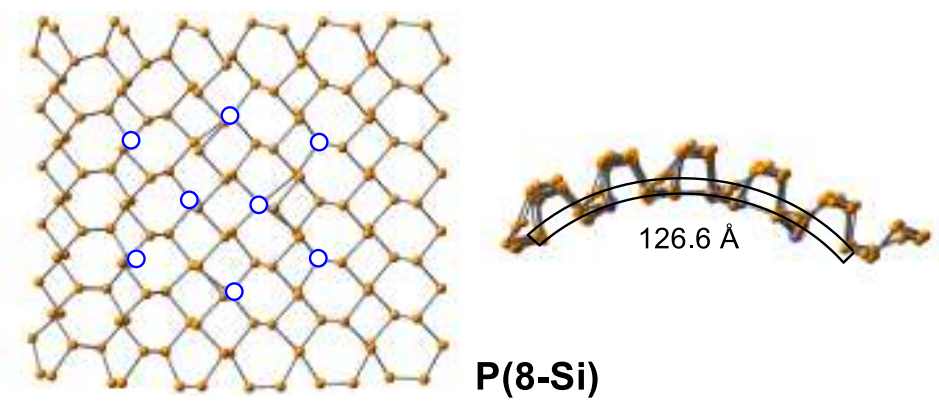

(d)
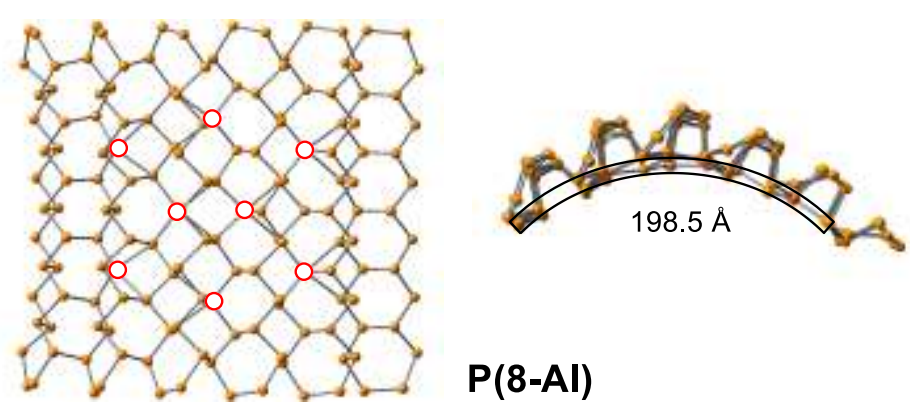

$P(8-A)$

Fig. 1 Optimized geometries and deviation from plane for (a) the pristine system and those substituted with 8 atoms of (b) sulfur highlighted in green, (c) silicon highlighted in blue, and (d) aluminum highlighted in red. The deviation from plane was determined in the plane containing the substituted atoms.

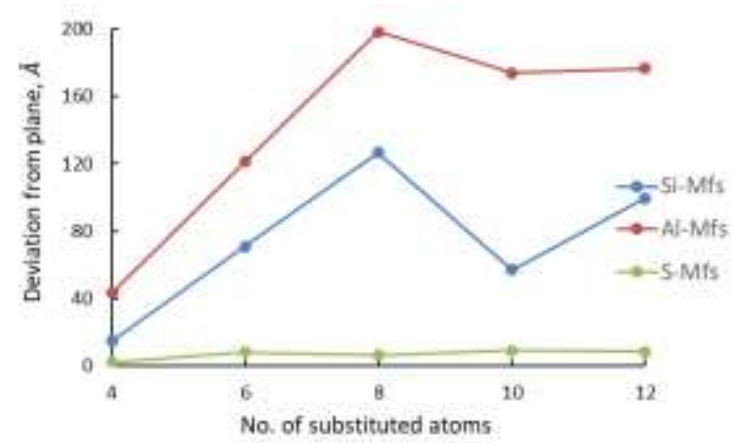

Fig. 2 Deviation from plane as a function of the number of doping atoms for Mfs under study.

Fig. 3 shows the optimized geometries of the pristine biflake structure, P-Bf, and the corresponding substituted systems with 16 atoms of $\mathrm{S}, \mathrm{Si}$, and $\mathrm{Al}$. It should be mentioned that there are three other possible 
stacking structures for P-Bf, which are less stable than that shown in Fig. 3 (See Fig. S2 in the ESI). For biflakes (Bfs) under study, the stability calculations reveal that almost all Bfs are closed-shell singlet. Only $\mathbf{B f}(\mathbf{1 2}-\mathbf{S}), \mathbf{B f}(\mathbf{2 0 - S}), \mathbf{B f}(\mathbf{2 4 - S )}$ and $\mathbf{B f ( 2 0 - A l )}$ presented instability of the wavefunction, hence they were computed as OSS systems. RASSCF calculations show that the singlet state is more stable than the triplet.

The deviation from plane values allow us to infer that the change in the planarity of the interacting layers depends on the type of replaced atom. For example, the deformation in Al-Bfs is due to the formation of intermolecular interactions of $\mathrm{Al}$ with nearby phosphorus atoms. It is also observed for Si-doped Bfs but to a lesser degree. As with monoflakes (Fig. 1), the order of planarity is the same, P-Bf $>$ S-Bfs $>$ Si-Bfs $>$ AlBfs. This effect extends for the systems of 8, 12, 20, and 24 heteroatoms, as observed in Fig. 4. Furthermore, the results indicate that the increase in substitution level leads to a decrease in planarity. In this case, for systems substituted with aluminium atoms, the deviation from plane ranges between 2.7-12.4 $\AA$, while the variation is less for Si and almost invariant for S-substituted complexes.

On the other hand, the interlaminar distance of Bfs also shows a clear trend (Fig. 3 and 5). If we consider the van der Waals radius, $r_{\mathrm{W}}$, of $\mathrm{P}\left(r_{\mathrm{W}}: 1.80 \AA\right)$ and doping atoms $\mathrm{Al}\left(r_{\mathrm{W}}: 1.84 \AA\right)$ and $\mathrm{Si}\left(r_{\mathrm{W}}: 2.10 \AA\right)$ as an interaction measure, the formation of interlaminar interactions Al-P and Si-Si as seen in Fig. 3 and Fig. S3 in the ESI leads to shorter interlaminar distances into Bfs. S-doped systems do not present interlaminar contacts because $\mathrm{S}\left(r_{\mathrm{W}}: 1.80 \AA\right.$ ) has the same $r_{\mathrm{W}}$ as the $\mathrm{P}$ atom. The presence of these bonds generates a distortion in the interacting phosphorene nanoflakes, which in the case of Al and Si is more noticeable (Fig. 4). Comparing all the systems (Fig. 5), the interlaminar distances for the 8 and 12 atom substitutions cause an increase of that length in comparison with P-Bf. This effect is maintained for all S-systems, but in the case of $\mathrm{Al}$ and $\mathrm{Si}$, the replacements involving 16, 20, and 24 atoms form biflakes of a smaller interlaminar distance than the $\mathbf{P}$ Bf as shown in Fig. 5. Al-Bfs exhibit the largest change in interlaminar distance that varies between $2.39 \AA$ and $2.67 \AA$. In contrast, the $S$ atom leads to a smaller variation that ranges from 2.57-2.74 $\AA$. These results can be explained from the geometries obtained in Fig. 3 and Fig. S3 in the ESI. As the level of substitution increases, the number of interlaminar contacts $\mathrm{Al}-\mathrm{P}$ and (Si-Si, Si-P) also increases. In contrast, in systems substituted with sulfur, the formation of interlaminar bonds is not observed. 


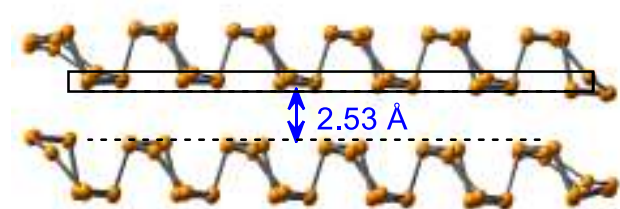

P-Bf

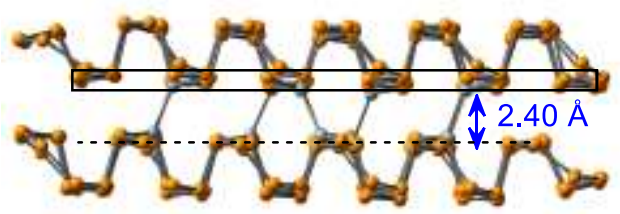

$\mathrm{Bf}(16-\mathrm{Si})$

8 interlaminar interactions:

Si-Si $(2.48-2.58 \AA)$

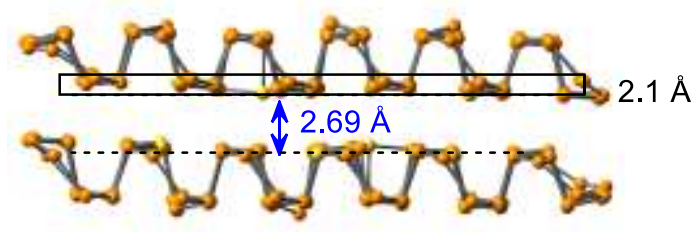

Bf(16-S)

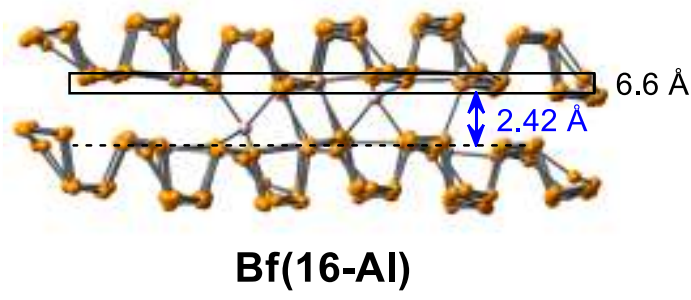

11 interlaminar interactions: Al-P (2.51-2.80 Å)

Fig. 3 Optimized geometries, deviation from plane and interlaminar distances of the pristine biflake, P-Bf, and those substituted with 16 atoms of S, Si or Al. Interlaminar interactions and their corresponding length range are indicated.

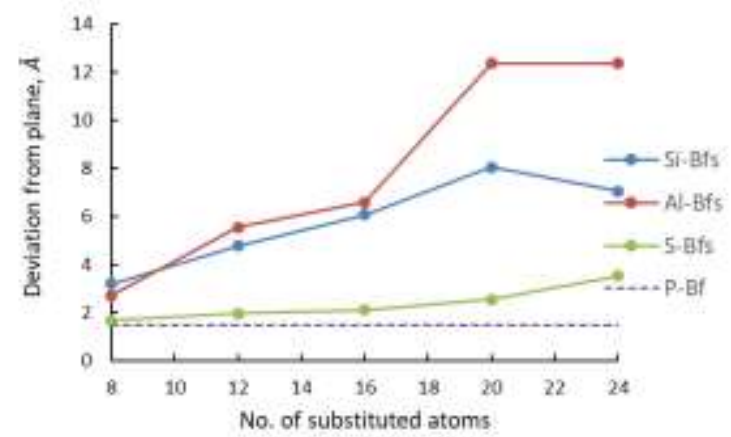

Fig. 4 Deviation from plane as function of the number of atoms substituted for Bfs under study.

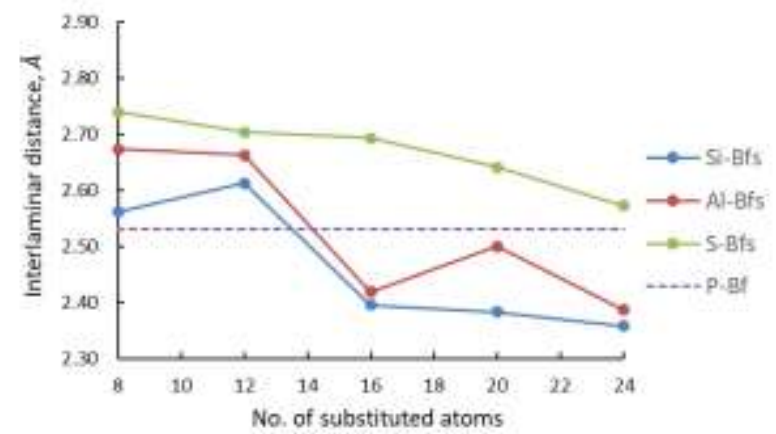

Fig. 5 Interlaminar distances as a function of the number of atoms substituted for Bfs studied. 
According to the analysed geometric parameters in both the monoflakes and biflakes, it can be assumed that the systematic substitution with $S$ atoms leads to small variations in phosphorene structure. In contrast, all heteroatoms generate considerable structural changes, and the case of systems with $\mathrm{Si}$, these have intermediate behaviour. This trend that maintains in the entire range of substitution can be explained in terms of the atomic radii. The sizes of the $\mathrm{S}$ and $\mathrm{P}$ atoms are similar, as is the case for $\mathrm{Al}$ and $\mathrm{Si}$. It means that $\mathrm{S}$ atoms do not produce such large structural changes on doping, whereas $\mathrm{Al}$ and Si substitutions exhibit comparable structural variations.

\section{Binding energies for biflakes}

The relative binding energies for Bfs are shown in Fig. 6. The energies values indicate that that silicon systems exhibit larger binding energies. In the case of aluminium, the inclusion of this type of atom in the phosphorene network shows a variation in binding energy like P-Bf. Meanwhile, S-BFs have lower formation energy than the other complexes. This trend may be consistent with the geometric parameters previously discussed.

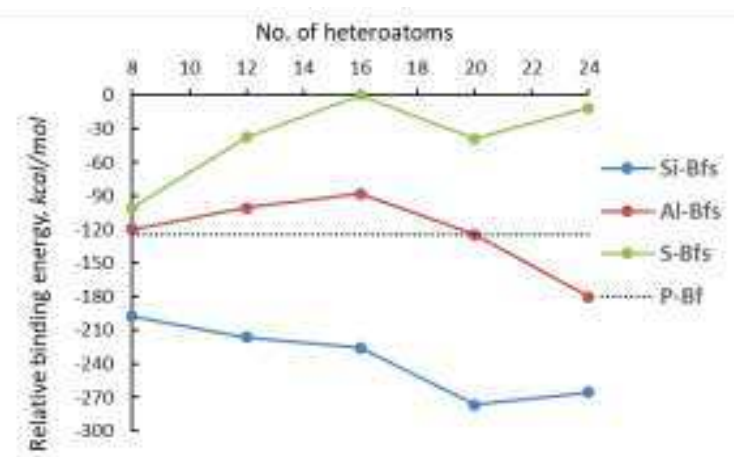

Fig. 6 Relative binding energies as a function of the number of heteroatoms substituted for BFs studied. The values are with respect to the system with the lowest interaction energy, $\mathbf{B f}(\mathbf{1 6 - S})$.

It is worth mentioning that as the number of heteroatoms increases, the binding energies tend to decrease, which applies to Al-Bfs and Si-Bfs. This energetic tendency can be explained due to interlaminar interactions Al-P, Si-Si, and Si-P previously discussed (Fig. 3 and Fig. S3 in the ESI). These contacts stabilize the complexes, whose effect is most noticeable for Si-Bfs since they mainly contain Si-Si interactions. While for complexes with $\mathrm{Al}$, the most numerous interlaminar contacts are Al-P. In both cases, the increase in substitution also rises the number of this kind of interaction. As a result, the binding energy decreases. In contrast, for complexes with $S$, the energy increases with the substitution degree, because in the $S$ systems the interlaminar interactions are not observed. 


\section{Electronic properties in mono- and biflakes}

Band gap

The band gap trends for Mfs are shown in Fig. 7(a). The doping with any of the heteroatoms, Al, Si, and S, generates a decrease in the band gap concerning the phosphorus nanoflake, P-Mf. The data also indicate that the $\mathrm{Al}$ atom inclusion generates a less significant decrease in band gap than silicon and sulfur. For sulfur complexes, this non-metal makes it possible to modify the phosphorene monolayer band gap from $0.02 \mathrm{eV}$ to $0.78 \mathrm{eV}$ with 4 and 6 doping heteroatoms respectively. Not only the nature of doping heteroatoms could explain these differences in the band gap, but also the structural change on phosphorene nanoflakes can alter the electronic properties. ${ }^{31}$ In the case of biflakes, the band gaps are displayed in Fig. 7(b). Values of those complexes, which are less than that exhibited by their corresponding monolayers (see Fig 7), are also altered by substituting with $\mathrm{Si}, \mathrm{Al}$, and $\mathrm{S}$. We also noted that the nature of band gap is affected by the type of substituted heteroatom. The inclusion of Al atoms decreases the semiconductor behavior of pristine phosphorene and, it is more altered with Si and S doping.

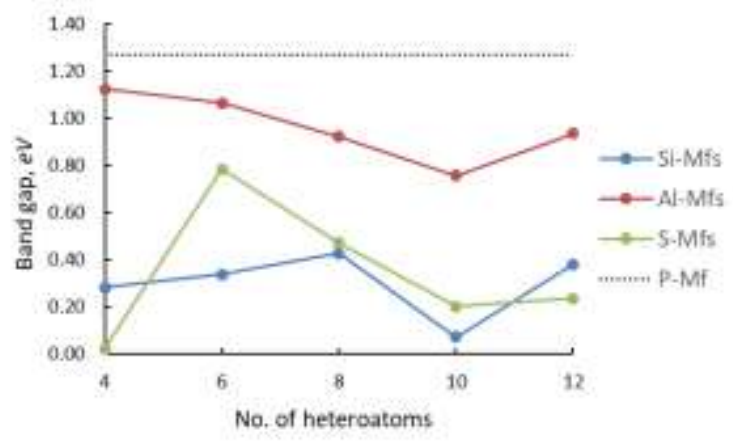

(a)

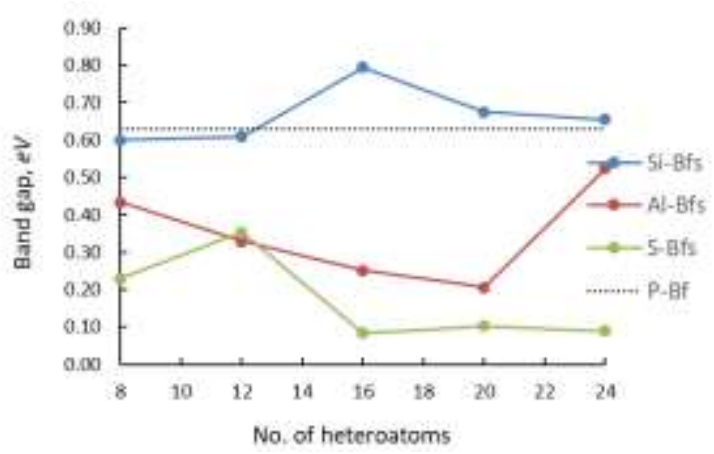

(b)

Fig. 7 Band gap values as a function of the number of heteroatoms substituted for (a) Mfs and (b) Bfs and studied.

For Si-Bfs, the band gap remains unchanged concerning the pristine complex as shown in Fig. 7(b). However, in the case of 16 aggregated heteroatoms, it generates a band gap opening of 0.63 to 0.79 eV taking P-Bf as a reference. In the case of $\mathrm{Al}$, the 8 and 24 heteroatom systems have the largest band gaps, which are 0.43 and $0.52 \mathrm{eV}$ respectively. For S-Bfs, increasing the number of heteroatoms in the phosphorene Bfs leads to a decrease in band gap to approximately $0.1 \mathrm{eV}$. The doping with $\mathrm{Si}$ atoms maintains the semiconductor character of $\mathbf{P}-\mathbf{B f}$ and, it is more distorted with $\mathrm{Al}$ and $\mathrm{S}$ substitution. These results allow us to suggest that the semiconductor character of Mfs and Bfs can be modified depending on the type of heteroatom used and its saturation in the system. Most of the studied species have closed ground state except $\mathbf{P ( 4 - S ) , P ( 4 - S i )}$ and $\mathbf{P ( 1 2 - S )}$, their closed-shell singlet states were found to be unstable and were reoptimized using broken symmetry method. The expectation values of $\left\langle S^{2}>\right.$ were found to be of $2.02,0.33$, and 0.03 , respectively. We have estimated the number of effectively unpaired electrons using the Head-Gordon formula. ${ }^{32}$

$$
\mathrm{N}_{\mathrm{u}}=\sum_{\mathrm{i}=1}^{\mathrm{M}} \mathrm{n}_{\mathrm{i}}^{2}\left(2-\mathrm{n}_{\mathrm{i}}\right)^{2}
$$


where $N_{u}$ is the number of effectively unpaired electrons, $n_{i}$ is the occupation of $i$-th natural orbital and $\mathrm{M}$ is the total number of active orbitals. According to RASSCF calculations, $\mathbf{P ( 4 - S )}$ and $\mathbf{P ( 4 - S i )}$ have 4 effectively unpaired electrons, each of them is located at the corresponding heteroatom (Fig. 8) while $\mathbf{P ( 1 2 -}$ S) has slightly less than one effectively unpaired electron. Therefore, $\mathbf{P ( 4 - S )}$ and $\mathbf{P ( 4 - S i )}$ have polyradicalic ground states while that of $\mathbf{P ( 1 2 - S )}$ is a nearly closed-shell singlet. As seen in Fig. 8, RASSCF description makes perfect sense. In $\mathbf{P}(\mathbf{4}-\mathrm{Si})$ an unpaired electron is a free valence of $\mathrm{Si}$ atom, an $s p^{3}$ orbital having one electron, while in the case of $\mathbf{P ( 4 - S )}$ unpaired electron is located at the $3 p$ orbital of $\mathbf{S}$ atom, an extra electron which is left after forming three bonds with neighboring phosphorus atoms. Notably, heavy substitution with sulfur destroys polyradicalic nature of ground states of substituted NFs due to the interaction between dopant atoms similar to Si substitution. ${ }^{21}$

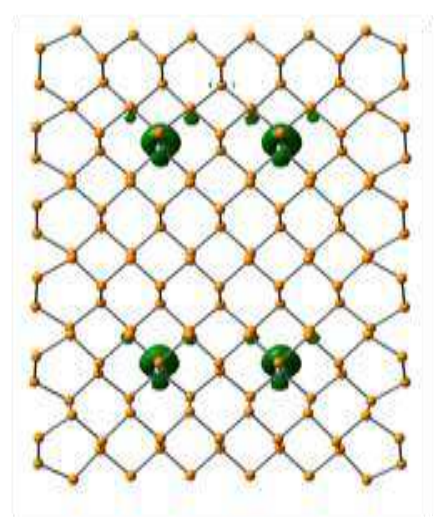

$\mathrm{P}(4-\mathrm{Si})$

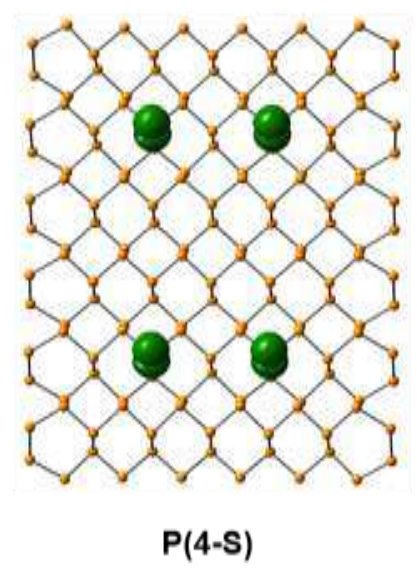

Fig. 8 Superposition of single occupied active orbitals for $\mathbf{P ( 4 - S )}$ and $\mathbf{P}(\mathbf{4}-\mathbf{S i})$ systems.

\section{Density of states}

Fig. 9 shows the total density of states (TD) for P-Mf and the most heavily doped Mfs, P(12-A1), P(12-Si), and P(12-S). According to Fig. 9, DOS spectra reproduce a similar trend of band gap values of highly substituted Mfs compared with HOMO-LUMO differences exhibited in Fig. 7(a). Nevertheless, both methods estimated a drop of band gap on doping, smaller for Al-Mfs, and larger for doping with Si and S. All this due to the absence and presence of an unpaired electron in Si-Mfs and S-Mfs, respectively. Similar effects are observed for Bfs complexes substituted with Al, however, Si-Bfs show unalter band gap data concerning P-Bf (Fig. 7(b) and Fig. S4 in the ESI). As in Mfs, the trend of band gap variation from DOS spectra also correspond to those observed in Fig. 7(b). 


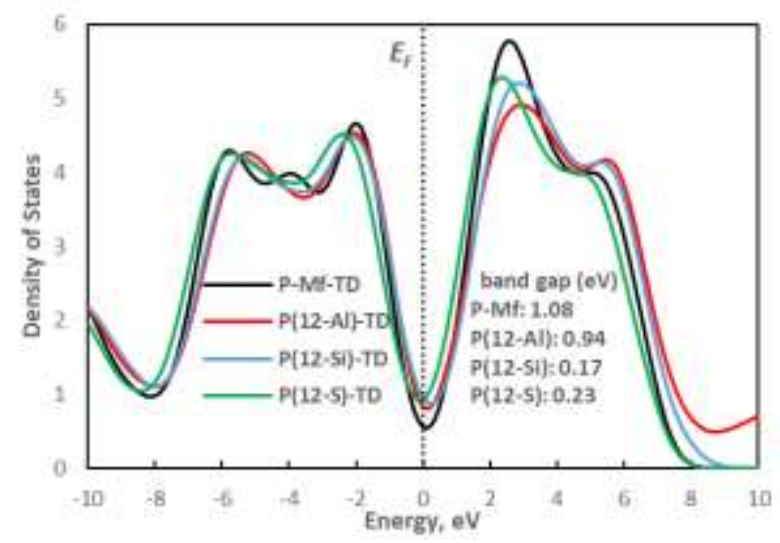

Fig. 9 Total (TD) density of states from TPSS calculations for pristine, P-Mf, and P(12-Al), P(12-Si) and P(12S). The band gap values are indicated.

\section{Ionization potentials (IP) and electron affinities (EA)}

The ionization potentials (IP) and the electron affinities (EA) trends for Mfs are shown in Fig. 10. The substitution process affects these two properties, regarding the pristine phosphorene, P-Mf. IP decreases slightly with the presence of $\mathrm{Al}(5.7-5.8 \mathrm{eV})$ and does not seem to depend on doping degree as shown in Fig. 10(a). The $\mathrm{Al}$ atom has a lower atomic ionization potential compared to $\mathrm{Si}, \mathrm{P}$ and $\mathrm{S}$ atoms. It generates a slight decrease in IP values concerning the P-Mf. In contrast, the EA values of all the substituted systems are higher than that shown by the P-Mf, Fig. 10(b). Due to $\mathrm{Al}$ and $\mathrm{P}$ atoms have a comparable EA, it causes that doping with $\mathrm{Al}$ has less impact on the EA, especially at low substitution degrees. A heavy Al doping produces an appreciable structural change (Fig. 1) due to the polar covalent character of Al-P bonds. The electron density is more concentrated at the P atoms in Al-Mfs regarding PMf, S-Mfs and Si-Mfs, leading to higher energy released by attaching an electron.

Silicon and sulfur doping modify both IP and EA. For Si-doped Mfs, they have an increase in EAs compare to PMf, which remains practically independent on the degree of substitution. In S-Mfs, the oscillation ranges between 3.4 and $3.8 \mathrm{eV}$ (Fig. 10(b)), following a behavior similar to that of Si-Mfs. For Si-Mfs and S-Mfs, an increase in EA in doping may be related to the polyradical character that these atoms confer on phosphorene nanoflakes.

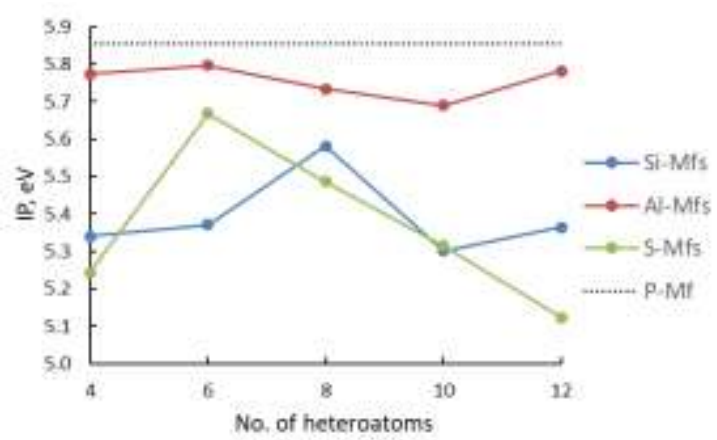

(a)

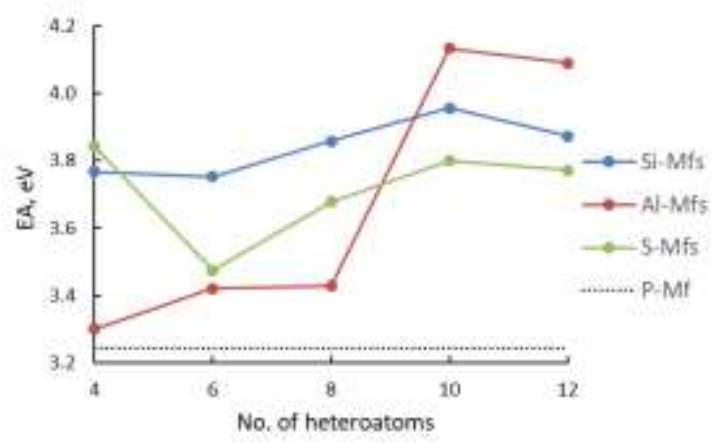

(b)

Fig. 10 Values of (a) IP and (b) EA, in eV, as a function of the number of heteroatoms in Mfs under study. 
The IP and EA results for Bfs studied are presented in Fig. 11. Computed data show that the IP increases or decreases considering P-Bf as a reference, which depends on the nature of the heteroatom. IP data decrease compared to monoflakes, while EA values remain almost in the same energy range (3.2 to $4.1 \mathrm{eV}$ ). IP values increase with the inclusion of $\mathrm{Al}$ and $\mathrm{Si}$ atoms, and it also increases with the number of heteroatoms from 5.3 to $5.6 \mathrm{eV}$ approximately (Fig. 11(a)). The behavior of the Al and Si systems could be explained by the formation of interlaminar bonds Al-P, Al-P, Si-P and Si-Si. It could suggest an alike electronic delocalization in Al-Bfs and Si-Bfs and therefore similar IP values. In contrast, the trend of S-Bfs is similar to its monolayer counterpart. $S$ atom is more electronegative than the $\mathrm{P}$ atom and it generates a decrease in the electron density in $\mathrm{P}$ atoms, decreasing the IP values of S-Bfs. Regarding the EA values (Fig. 11 (b)), the results are similar to those exhibited by doped monoflakes (Fig. 10 (b)), which follows that stacking does not affect this property.

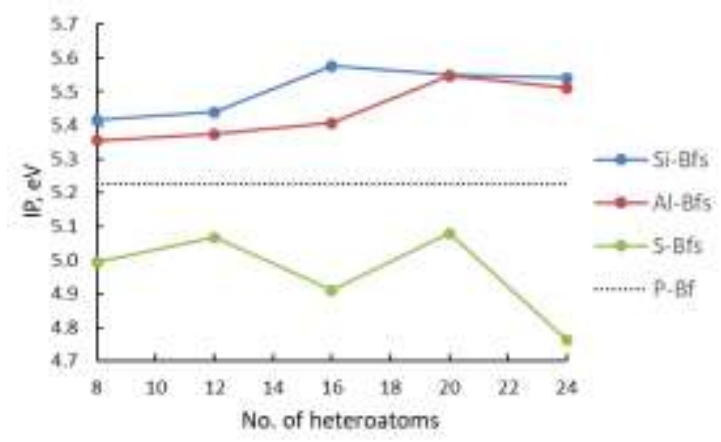

(a)

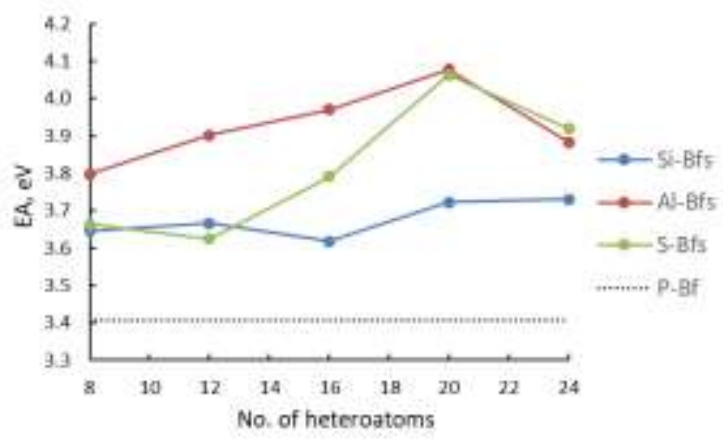

(b)

Fig. 11 Values of (a) IP and (b) EA, in eV, as a function of the number of heteroatoms in biflakes under study.

\section{NBO charges and reorganization energies}

Fig. 12(a) and 12(b) show the sum of NBO charges of the phosphorene skeleton and heteroatoms present into the Bfs, respectively. For Al-Bfs and Si-Bfs (Fig. 12(a)), the charge of the phosphorene scaffold is more negative as the degree of substitution increases, which is due to Al and Si atoms are less electronegative than P. For S-Bfs, the natural charge of the phosphor network becomes more positive as the number of heteroatoms increases. This is because the electronegative nature of $\mathrm{S}$ compared to the P atom. On the other hand, the sum of the heteroatom charges (Fig 12(b)) is opposite to that observed with the phosphorene skeleton. These two types of charge offset each other. The variation observed for the three systems corresponds to the order of the electronegativity S > P > Si > Al. Similar results are observed for Mfs (Fig. S5 in the ESI). 


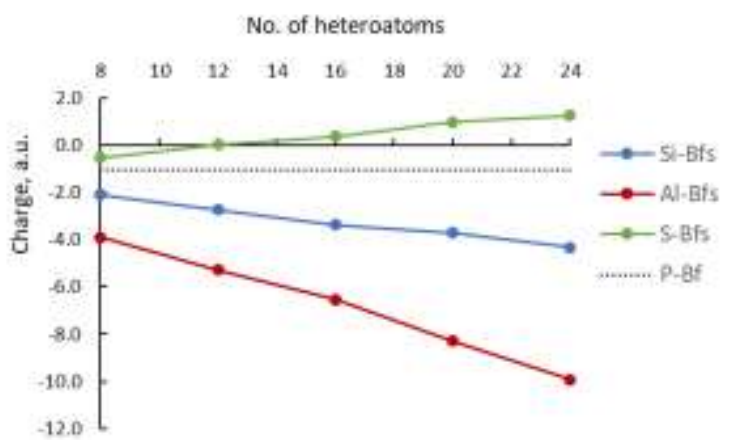

(a)

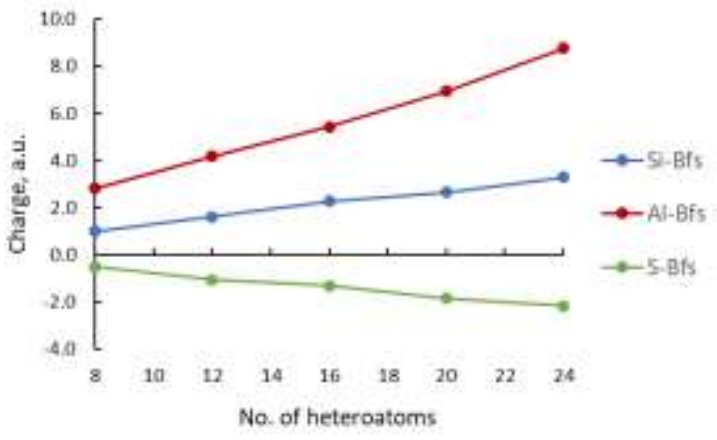

(b)

Fig. 12 Sum of the NBO charges of (a) the phosphorene skeleton and (b) heteroatoms involved in Bfs.

Finally, hole and electron reorganization energies for Mfs are shown in Table 1. The divergence in magnitude that exists between the hole reorganization energy $\left(\lambda_{+}\right)$and the electron reorganization energy $\left(\lambda_{-}\right)$ represents that the delocalization of the positive and negative charge is not the same. Regarding $\lambda_{+}$energies, the substitution with $\mathrm{Al}$ increases $\lambda_{+}$concerning P-Mf, although it only decreases for $\mathbf{P}(\mathbf{1 2}-\mathbf{A l})$. Doping with Al generates that $\lambda_{+}>\lambda_{-}$means that Al-Mfs are the hole transport materials. It is the same for Si-Mfs at the low substitution level, however, the higher doping with Si leads to the electron transport materials. In systems doped with S, the substitution practically retains the $\lambda_{+}$and $\lambda_{-}$values unchanged concerning P-Mf.

Table 1. Hole $\left(\lambda_{+}\right)$and electron $\left(\lambda_{-}\right)$reorganization energies as a function of the number of heteroatoms substituted for Mfs and Bfs under study.

\begin{tabular}{lccc|ccc}
\hline & \multicolumn{3}{c}{$\lambda_{+}$} & \multicolumn{3}{c}{$\lambda_{-}$} \\
\cline { 2 - 7 } Monoflakes & \multicolumn{5}{c}{$(\mathrm{eV})$} \\
\cline { 2 - 7 } & $\mathrm{Si}$ & $\mathrm{Al}$ & $\mathrm{S}$ & $\mathrm{Si}$ & $\mathrm{Al}$ & $\mathrm{S}$ \\
\hline P(4-at) & 0.13 & 0.18 & 0.14 & 0.17 & 0.26 & 0.14 \\
P(6-at) & 0.17 & 0.18 & 0.11 & 0.25 & 0.27 & 0.12 \\
P(8-at) & 0.14 & 0.17 & 0.12 & 0.28 & 0.44 & 0.17 \\
P(10-at) & 0.28 & 0.17 & 0.12 & 0.21 & 1.09 & 0.14 \\
P(12-at) & 0.24 & 0.10 & 0.14 & 0.22 & 1.05 & 0.47 \\
\hline P-Mf & & 0.14 & & & 0.12 \\
\hline
\end{tabular}

\begin{tabular}{lccc|ccc}
\hline & \multicolumn{4}{c}{$\lambda_{+}$} & \multicolumn{4}{c}{$\lambda_{-}$} \\
\cline { 2 - 7 } Biflakes & \multicolumn{4}{c}{$(e V)$} \\
\cline { 2 - 7 } & $\mathrm{Si}$ & $\mathrm{Al}$ & $\mathrm{S}$ & $\mathrm{Si}$ & $\mathrm{Al}$ & $\mathrm{S}$ \\
\hline Bf(8-at) & 0.04 & 0.06 & 0.06 & 0.15 & 0.36 & 0.24 \\
Bf(12-atom) & 0.04 & 0.08 & 0.42 & 0.15 & 0.23 & 0.09 \\
Bf(16- atom) & 0.04 & 0.09 & 0.25 & 0.18 & 0.08 & 0.14 \\
Bf(20- atom) & 0.04 & 0.13 & 0.07 & 0.28 & 0.19 & 0.09 \\
Bf(24- atom) & 0.06 & 0.09 & 0.16 & 0.24 & 0.38 & 0.14 \\
\hline P-Bf & \multicolumn{3}{c|}{0.04} & & 0.10 \\
\hline
\end{tabular}

For biflakes, both $\lambda_{+}$and $\lambda_{-}$of the three sets studied are larger than or equal to those observed for P-Bfs. As for hole reorganization energies, Si substitution conserves the $\lambda_{+}$value almost unchanged compared to $\mathbf{P - B f}$. The doping with $\mathrm{Al}$ raises $\lambda_{+}$energies with respect to P-Bfs. In the specific case of sulfur, it shows an increase in the replacement of 12 and $16 \mathrm{~S}$ atoms, while at the lowest or highest degree of doping, the values are comparable to those exhibited by $\mathrm{Al}$ and $\mathrm{Si}$. This jump could be due to the OSS character of the species Bf(12-S), which would considerably alter its electron distribution and the properties derived from it. For 
pristine biflake P-Bf $\lambda_{-}>\lambda_{+}$, which agrees with the experimental observation about the high hole mobility that exhibits the phosphorene. ${ }^{11}$ According to our results (Table 1), Al-Bfs and Si-Bfs can also be considered as hole transport materials. For S-Bfs, there have been identified no clear correlation between the doping level and reorganization energies. The maximum $\lambda_{-}$is observed for $\mathbf{B f}(\mathbf{8}-\mathbf{S})$ and the highest $\lambda_{+}$for $\mathbf{B f}(\mathbf{1 2}-\mathbf{S})$.

\section{Conclusions}

The substitution of phosphorene monoflakes and biflakes with the $\mathrm{Al}, \mathrm{Si}$, and $\mathrm{S}$ heteroatoms have been studied from a theoretical perspective considering structural aspects and their electronic properties. In the case of monoflakes, the substitution with Al leads to significant variations at the structural level. Despite this, the variation of the band gap and IP magnitudes with the level substitution is not very large, always decreasing the value concerning the unsubstituted species, P-Mf. In the case of EA, its magnitude is more altered with the Al-doping level in comparison with the P-Mfs. Furthermore, Al also improves the hole mobility in the phosphorene nanoflake when the substitution is high. The Si atoms generate intermediate structural variations between $\mathrm{Al}$ and $\mathrm{S}$ in phosphorene monolayers. The substitution with $\mathrm{S}$ atoms does not significantly alter the structure of the phosphorene nanoflake. The doping with Si and S leads to a decrease in the band gap and IP concerning P-Mf. EAs and reorganization energies vary a similar way as the substitution degree decreases or increases, having both Si-Mfs and S-Mfs a similar behavior.

Regarding studied biflakes, the structural parameters show a similar trend to that observed for monoflakes. In the specific case of Al-Bfs and Si-Bfs, the appearance of interlaminar interactions Al-P, Si$\mathrm{Si}$, and $\mathrm{Si}-\mathrm{P}$ generates that the binding energies are considerably higher compared to those exhibited by $\mathrm{S}$ Bfs. Unlike its monolayer counterpart, the Si-doping does not change the band gap compared to the pristine bilayer P-Bfs but produces hole transport materials. Additionally, Si- and Al-substitution leads to an increase in IP concerning P-Bf, which occurs over the entire range of analyzed substitution. It could be explained by intermolecular interactions presented in those Bfs. For EA values, all doped biflakes follow a similar trend to their monolayer counterparts. In the case of reorganization energies, Al-complexes show high hole mobility whereas for S-Bfs no clear correlation is identified between the doping level and the reorganization energies. Our results indicate that the semiconductor character of Mfs and Bfs can be altered depending on the type of heteroatom used and its saturation in the system. Finally, the NBO charges reveal that the phosphorene skeleton and the sum of heteroatoms present in Mfs and Bfs follow a complementary trend. It obeys the order of electronegativity of $\mathrm{Al}, \mathrm{Si}, \mathrm{P}$ and $\mathrm{S}$ atoms. These computed data may be theoretical support for the development of doped phosphorene materials.

\section{Acknowledgements}

W.E.V.N. acknowledges support from DGAPA of the UNAM under postdoctoral fellowship Grant No. CJIC/CTIC/4732/2O2O. Cesar Gabriel Vera de la Garza acknowledges doctoral fellowship grant 859569 from CONACyT. 


\section{Declarations}

Funding information: We acknowledge the financial support from PAPIIT (IN201219/31) and the financial support from CONACyT (Grant 251684).

Conflicts of interest: The authors declare that they have no conflict(s) of interest.

Availability of data and material: N/A

Code availability: N/A

Authors' contributions: All authors contributed equally to this work, and all agree with the contents of the manuscript.

\section{References}

1 K. S. Novoselov, A. K. Geim, S. V. Morozov, D. Jiang, M. I. Katsnelson, I. V. Grigorieva, S. V. Dubonos, A. A. Firsov . Nature. 438, 197-200 (2005)

2 Y. Zhang, Y.-W. Tan, H. L. Stormer, P. Kim. Nature. 438, 201-204 (2005)

3 C. Berger, Z. Song, X. Li, X. Wu, N. Brown, C. Naud, D. Mayou, T. Li, J. Hass, A. N. Marchenkov, E. H. Conrad, P. N. First, W. A. de Heer. Science. 312, 1191-1196 (2006)

4 A. H. Castro Neto, F. Guinea, N. M. R. Peres, K. S. Novoselov, A. K. Geim. Rev. Mod. Phys. 81, 109-162 (2009)

5 S. Stankovich, D. A. Dikin, G. H. B. Dommett, K. M. Kohlhaas, E. J. Zimney, E. A. Stach, R. D. Piner, S. T. Nguyen, R. S. Ruoff. Nature. 442, 282-286 (2006)

6 R. R. Nair, P. Blake, A. N. Grigorenko, K. S. Novoselov, T. J. Booth, T. Stauber, N. M. R. Peres A. K. Geim. Science. 320, 1308 (2008)

7 A. C. Ferrari, J. C. Meyer, V. Scardaci, C. Casiraghi, M. Lazzeri, F. Mauri, S. Piscanec, D. Jiang, K. S. Novoselov, S. Roth, A. K. Geim. Phys. Rev. Lett. 97, 187401 (2006)

8 A. K. Geim K. S. Novoselov. Nature Mater. 6, 183-191 (2007)

9 P. R. Wallace. Phys. Rev. 71, 622-634 (1947)

10 E. S. Reich. Nature. 506, 19 (2014)

11 H. Liu, A. T. Neal, Z. Zhu, Z. Luo, X. Xu, D. Tomanek, P. D. Ye. Nano Lett. 8, 4033-4041 (2014)

12 L. Li, Y. Yu, G. J. Ye, Q. Ge, X. Ou, H. Wu, D. Feng, X. H. Chen, Y. Zhang. Nature Nanotech. 9, $372-377$ (2014)

13 J. Qiao, X. Kong, Z.-X Hu, F. Yang, W. Ji. Nature Commun. 5, 4475 (2014)

14 Z. Zhu D. Tomanek, Phys. Rev. Lett. 112, 176802 (2014)

15 A. Castellanos-Gomez. J. Phys. Chem. Lett. 6, 4280-4291 (2015)

16 Y. Jing, Q. Tang, P. He, Z. Zhou, P. Shen, Nanotechnology. 26, 095201 (2015)

17 D.A. Ospina, C.A. Duque, J.D. Correa, E.S. Morell. Superlattices Microstruct. 97, 562-568 (2016)

18 J. Guan, Z. Zhu, D. Tománek. Phys. Rev. Lett. 113, 046804 (2014) 
19 Z. Zhu, D. Tománek. Phys. Rev. Lett. 112, 176802 (2014)

20 E. Martínez Olmeda, C. G. Vera, S. Fomine. Comput. Theor. Chem. 1130, 33-45 (2018)

21 T. Gorkan, Y. Kadioglu, O. Üzengi Aktürk, G. Gökoğlu, E. Aktürk, S. Ciraci. J. Phys. Chem. C. 123, 30704-30713 (2019)

22 E. M. Olmedo, C. G.V. de la Garza, S. Fomine. J. Mol. Model. 25, 292 (2019)

23 J. Tao, J. Perdew, V. Staroverov, G. Scuseria. Phys. Rev. Lett. 91, 146401 (2003)

24 V. N. Staroverov, G. E. Scuseria, J. Tao, Jianmin, J. P. Perdew. Phys. Rev. B. 69, 075102 (2004)

25 TURBOMOLE V7.2 2017, a development of University of Karlsruhe and Forschungszentrum Karlsruhe GmbH, 19892007, TURBOMOLE GmbH, since 2007; available from http://www.turbomole.com.

26 M. J. Frisch, G. W. Trucks, H. B. Schlegel, G. E. Scuseria, M. A. Robb, J. R. Cheeseman, G. Scalmani, V. Barone, G. A. Petersson, H. Nakatsuji, X. Li, M. Caricato, A. V. Marenich, J. Bloino, B. G. Janesko, R. Gomperts, B. Mennucci, H. P. Hratchian, J. V. Ortiz, A. F. Izmaylov, J. L. Sonnenberg, D. Williams-Young, F. Ding, F. Lipparini, F. Egidi, J. Goings, B. Peng, A. Petrone, T. Henderson, D. Ranasinghe, V. G. Zakrzewski, J. Gao, N. Rega, G. Zheng, W. Liang, M. Hada, M. Ehara, K. Toyota, R. Fukuda, J. Hasegawa, M. Ishida, T. Nakajima, Y. Honda, O. Kitao, H. Nakai, T. Vreven, K. Throssell, J. A. Montgomery, Jr., J. E. Peralta, F. Ogliaro, M. J. Bearpark, J. J. Heyd, E. N. Brothers, K. N. Kudin, V. N. Staroverov, T. A. Keith, R. Kobayashi, J. Normand, K. Raghavachari, A. P. Rendell, J. C. Burant, S. S. Iyengar, J. Tomasi, M. Cossi, J. M. Millam, M. Klene, C. Adamo, R. Cammi, J. W. Ochterski, R. L. Martin, K. Morokuma, O. Farkas, J. B. Foresman, and D. J. Fox, Gaussian, Inc., Wallingford CT, 2016. Gaussian 16, Revision A.03.

27 R. Pablo-Pedro, H. Lopez-Rios, S. Fomine M. S. Dresselhaus. J. Phys. Chem. Lett. 8, 615-620 (2017)

28 T. Lu, F. Chen, Multiwfn: A Multifunctional Wavefunction Analyzer. J. Comput. Chem. 33, 580-592 (2012); Z. Liu, T. Lu, Carbon. 165, 461-467 (2020)

29 A. E. Torres, R. Flores S. Fomine. Synth. Met. 213, 78-87 (2016)

30 B.C. Lin, C.P. Cheng, Z.P.M. Lao. J. Phys. Chem. A. 107, 5241-5251 (2003)

31 M. Yeganeh, F. Badieian Baghsiyahi, R. Pilevar Shahri. Applied Physics A. 125, 545 (2019)

32 M. Head-Gordon. Chem. Phys. Lett. 372, 508-511 (2003) 
Figures

(a)
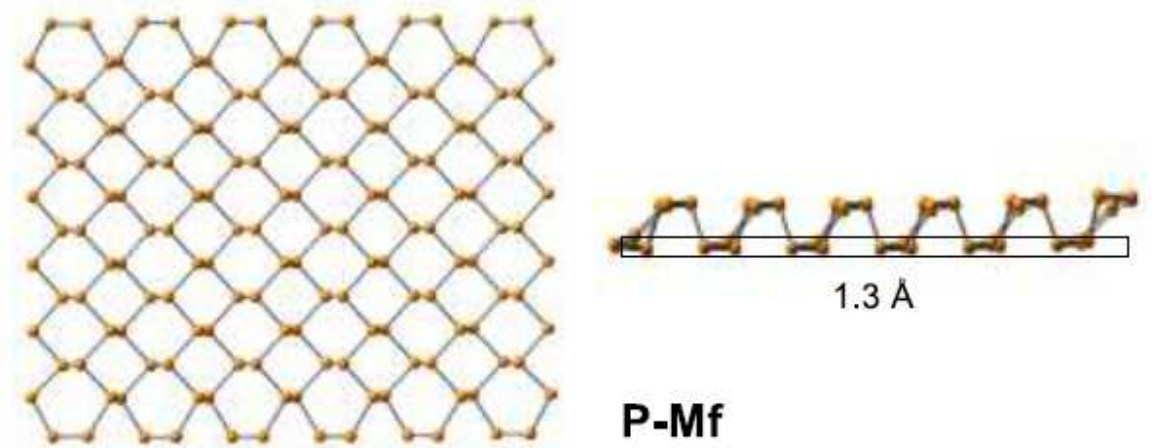

$1.3 \AA$

\section{P-Mf}

(b)
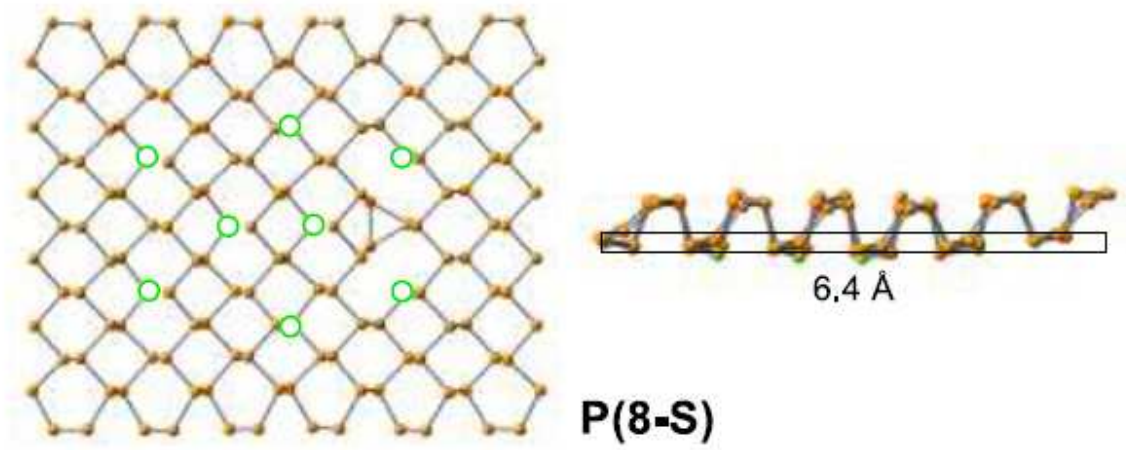

$\mathbf{P}(\mathbf{8 - S )}$

(c)
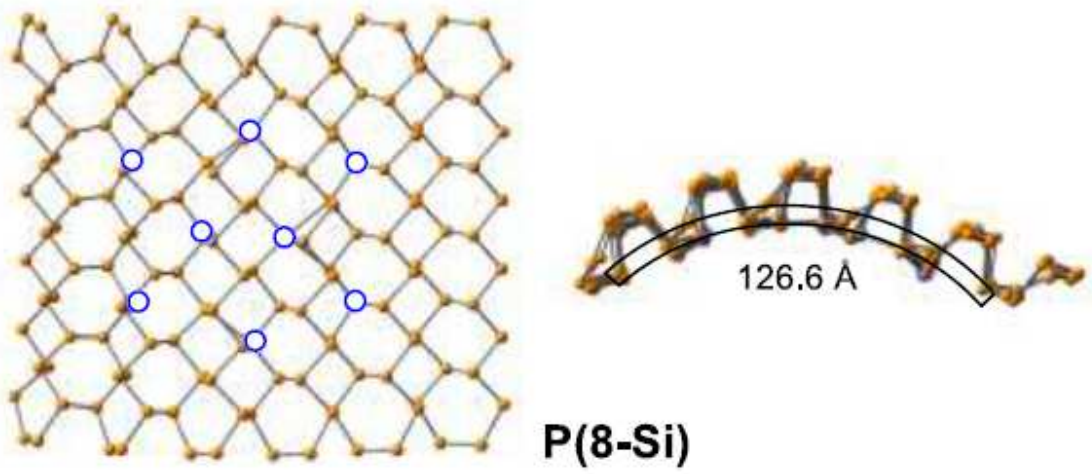

(d)

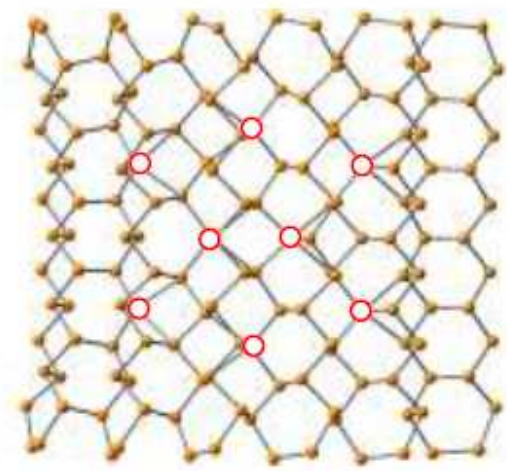

$\mathrm{P}(\mathrm{8}-\mathrm{Si})$

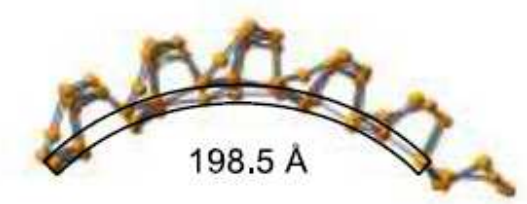

$P(8-A I)$

\section{Figure 1}

Optimized geometries and deviation from plane for (a) the pristine system and those substituted with 8 atoms of (b) sulfur highlighted in green, (c) silicon highlighted in blue, and (d) aluminum highlighted in red. The deviation from plane was determined in the plane containing the substituted atoms. 


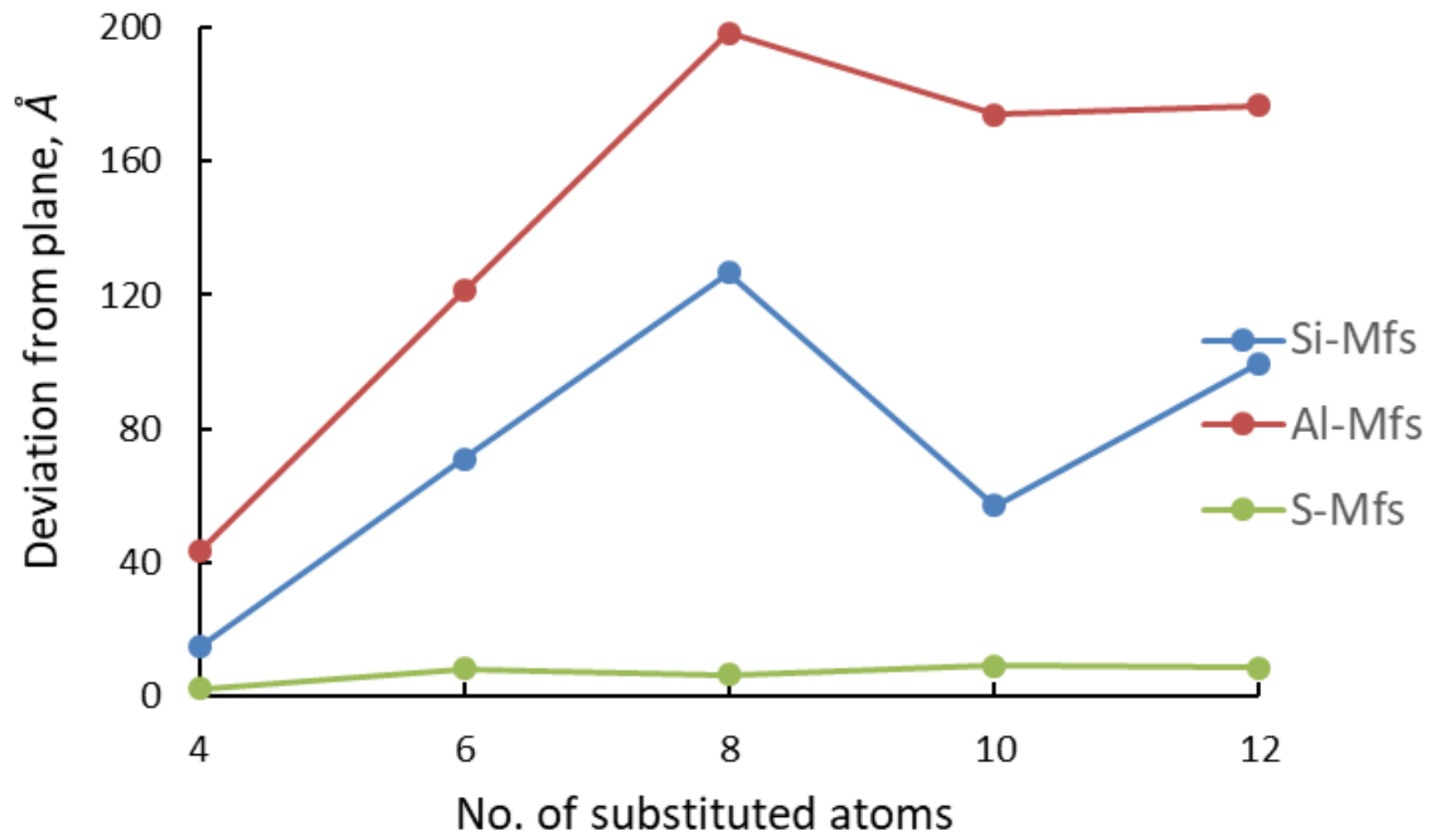

Figure 2

Deviation from plane as a function of the number of doping atoms for Mfs under study. 

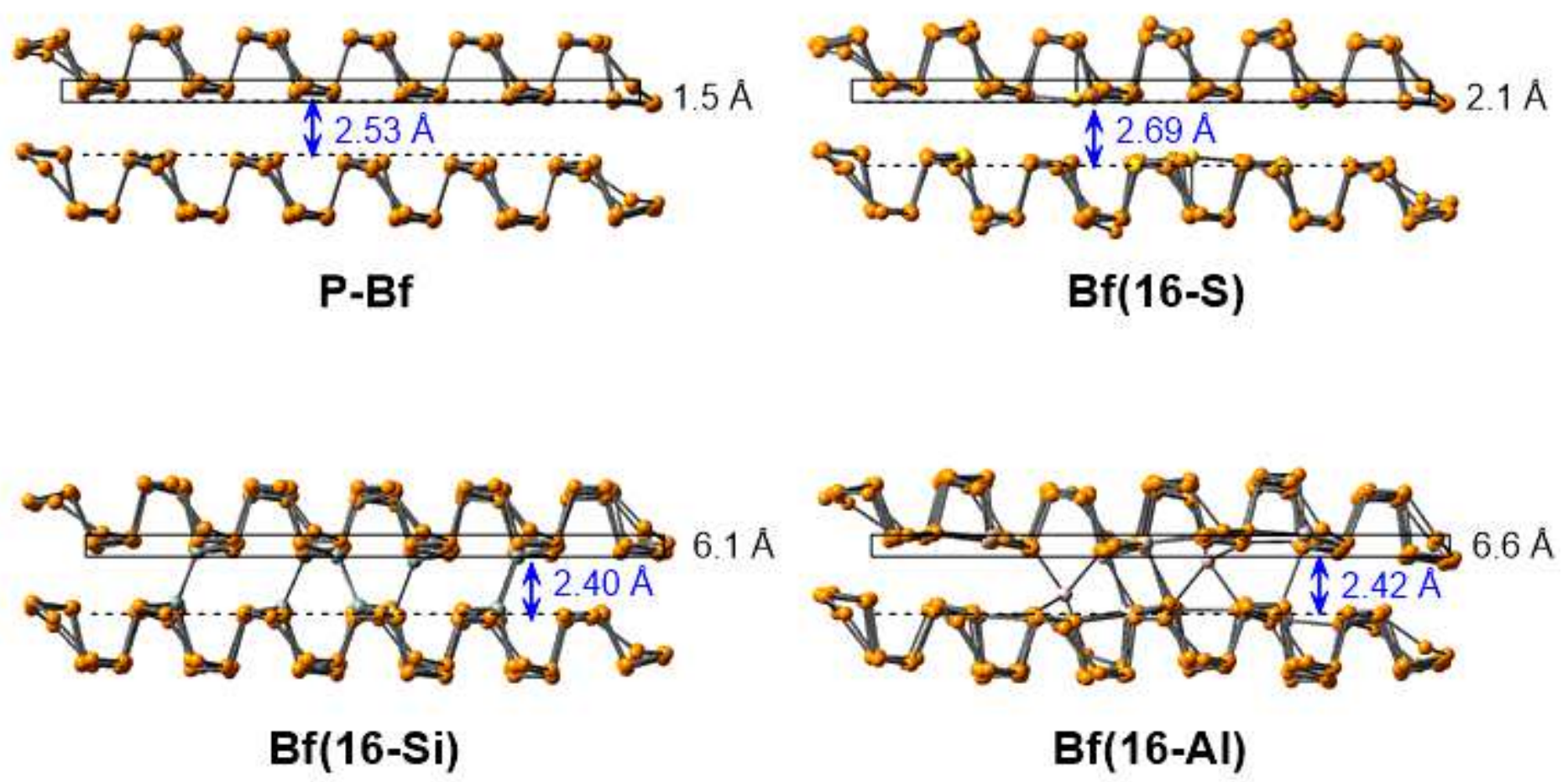

8 interlaminar interactions:

Si-Si $(2.48-2.58$ A)

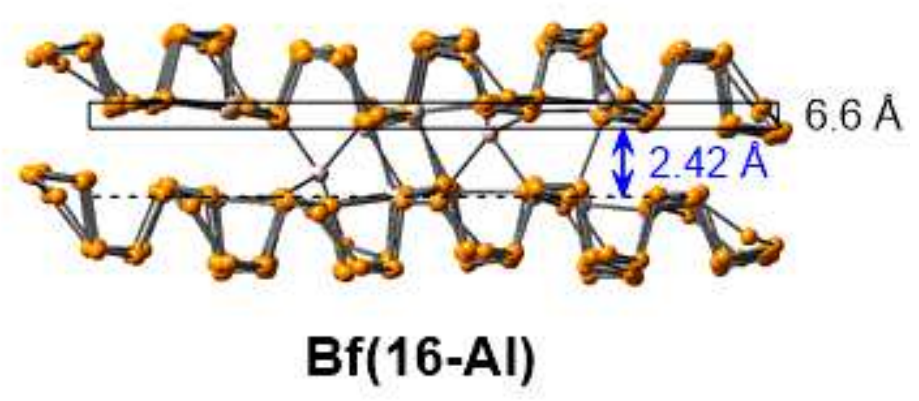

11 interlaminar interactions:

Al-P (2.51-2.80 Â)

\section{Figure 3}

Optimized geometries, deviation from plane and interlaminar distances of the pristine biflake, $\mathrm{P}-\mathrm{Bf}$, and those substituted with 16 atoms of S, Si or Al. Interlaminar interactions and their corresponding length range are indicated. 


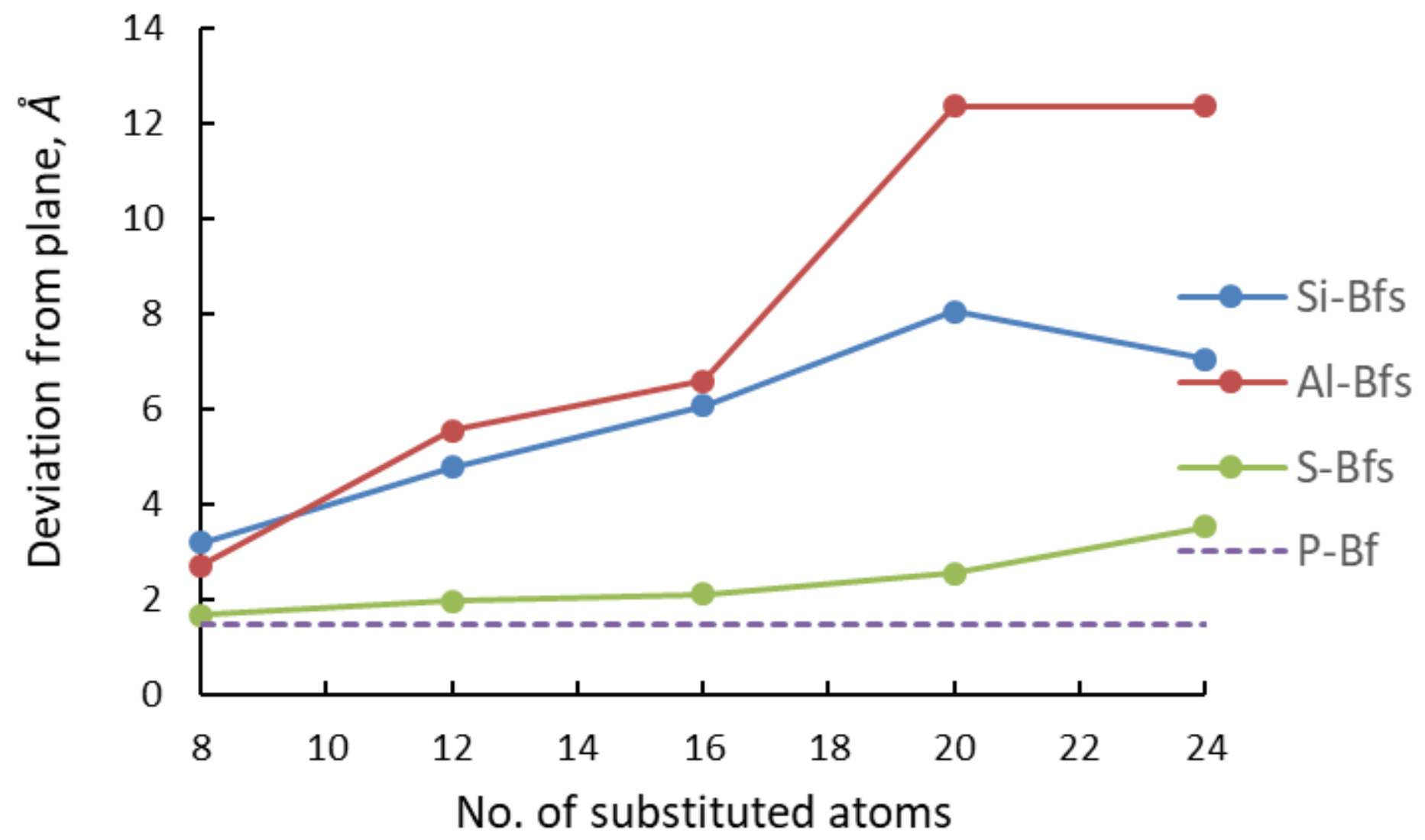

Figure 4

Deviation from plane as function of the number of atoms substituted for Bfs under study. 


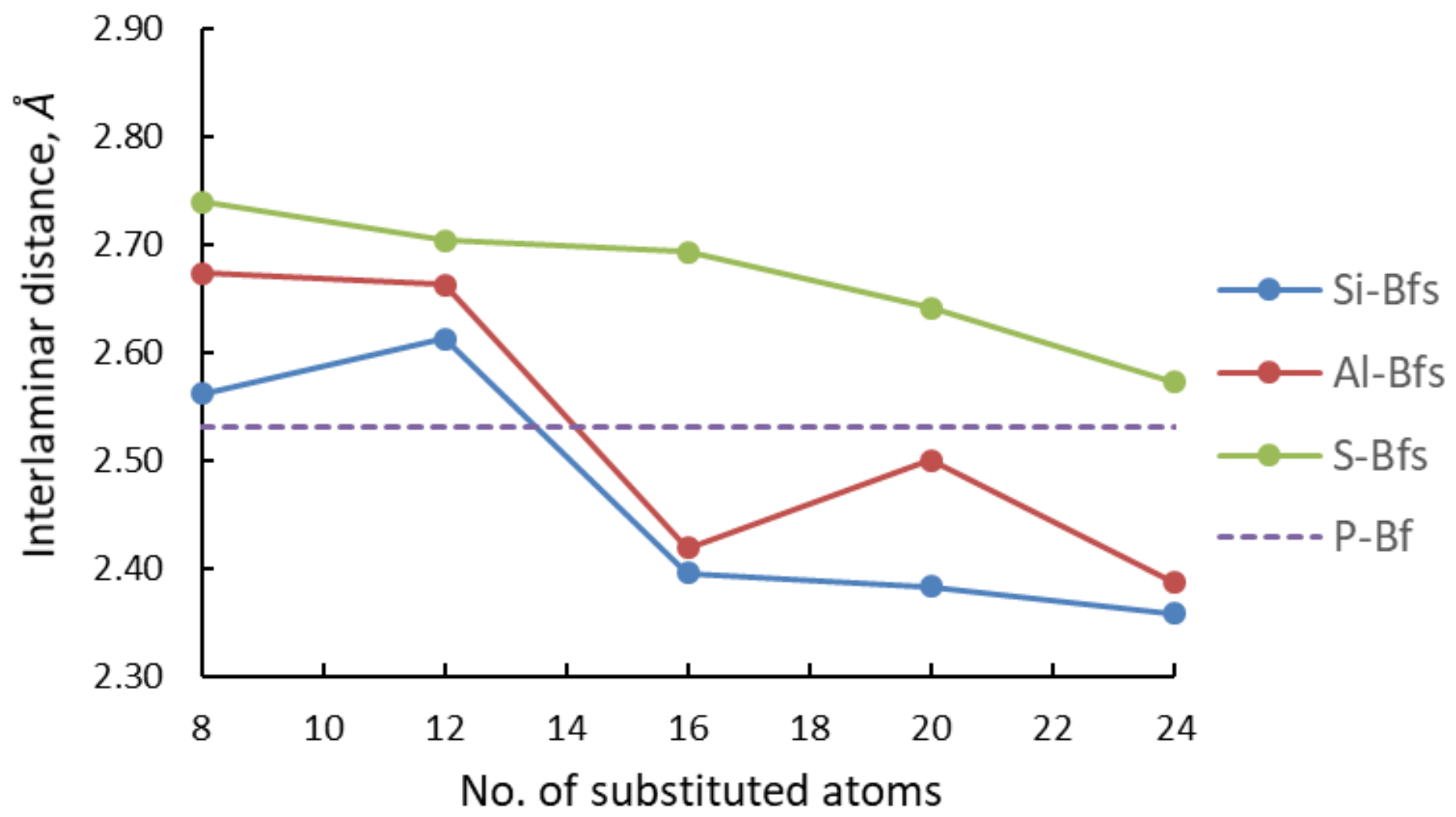

Figure 5

Interlaminar distances as a function of the number of atoms substituted for Bfs studied. 
No. of heteroatoms

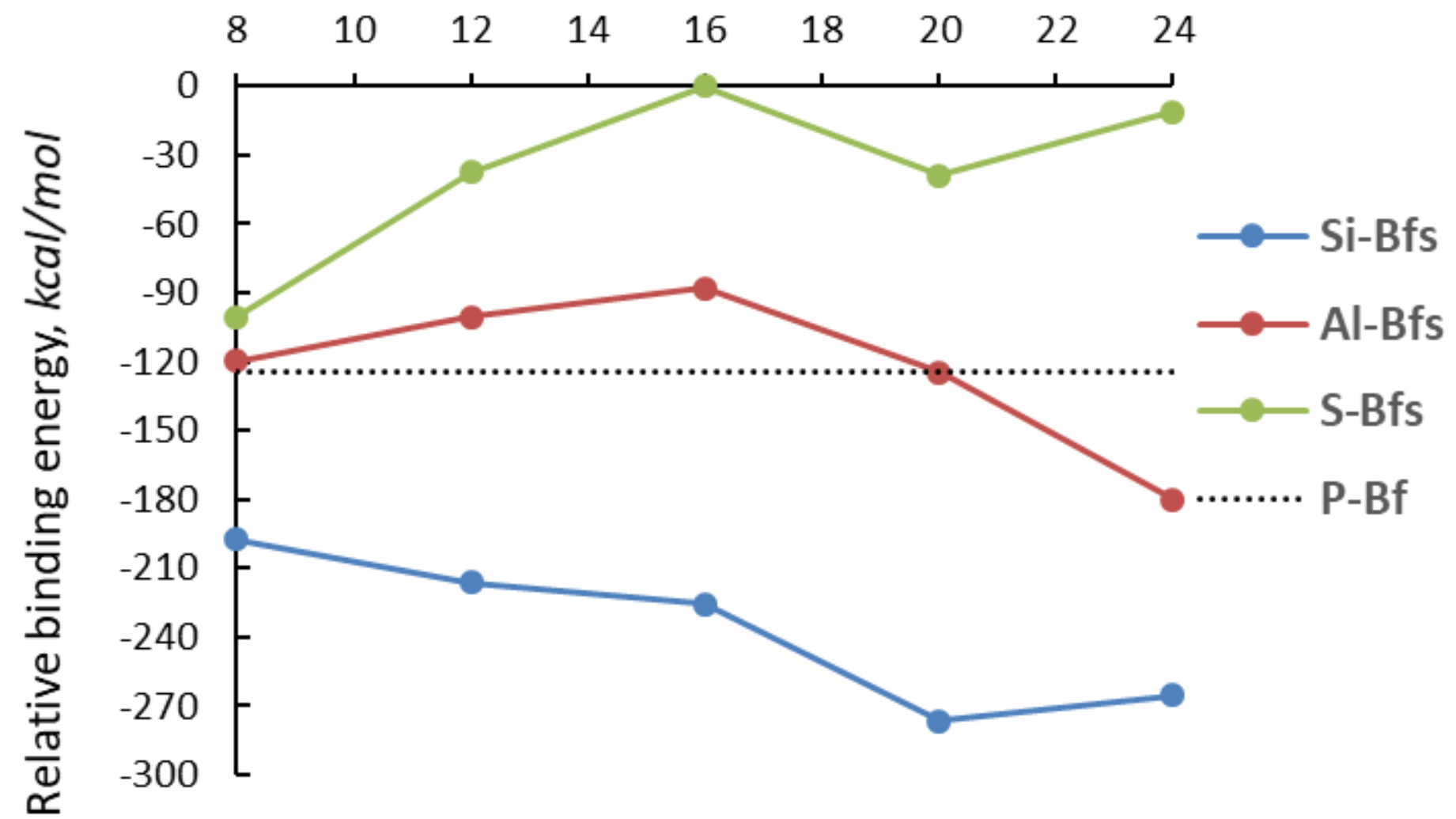

Figure 6

Relative binding energies as a function of the number of heteroatoms substituted for BFs studied. The values are with respect to the system with the lowest interaction energy, $\mathrm{Bf}(16-\mathrm{S})$.
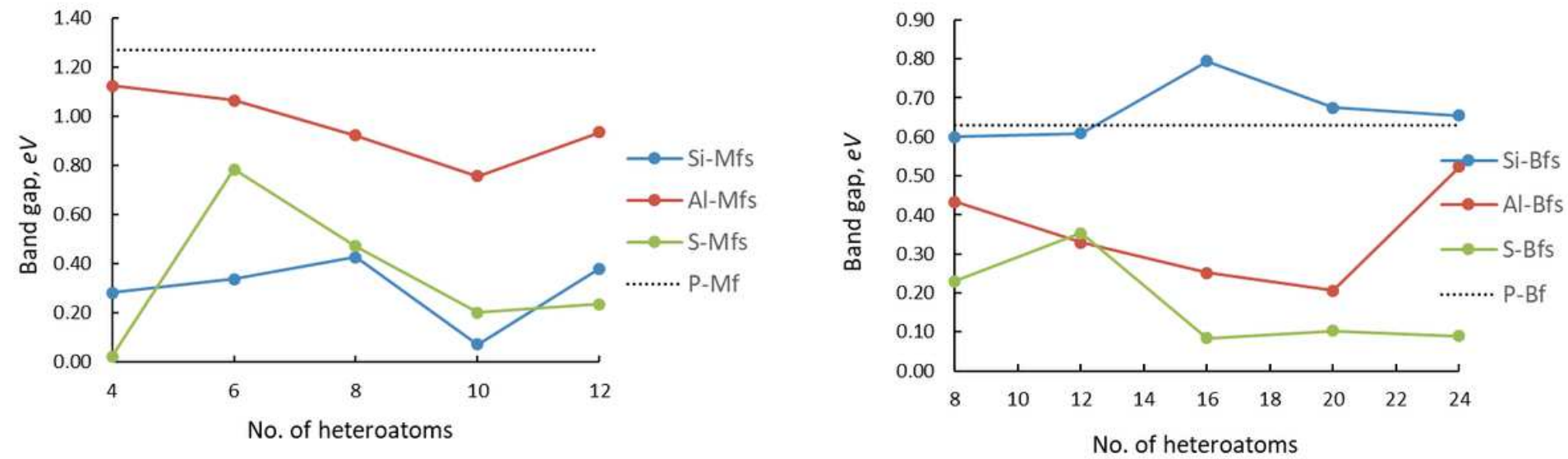

Figure 7

Band gap values as a function of the number of heteroatoms substituted for (a) Mfs and (b) Bfs and studied. 


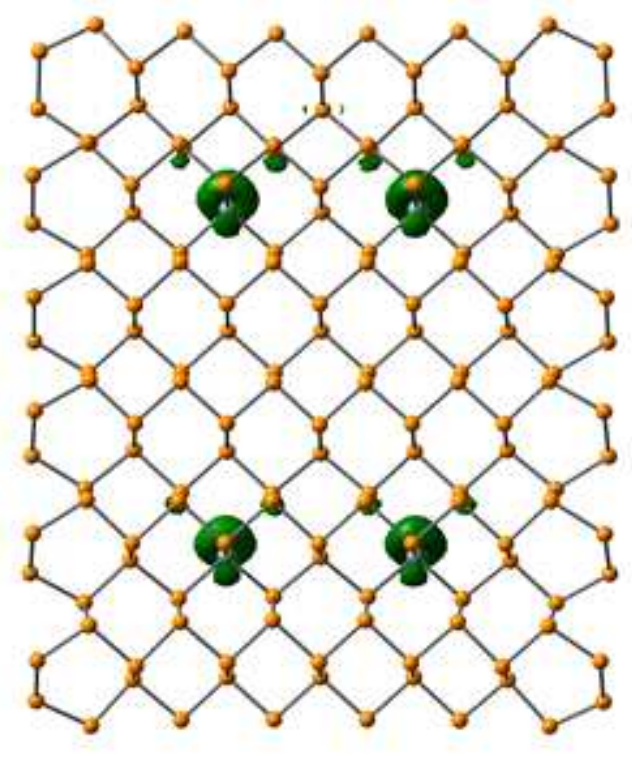

$\mathbf{P}(4-\mathrm{Si})$

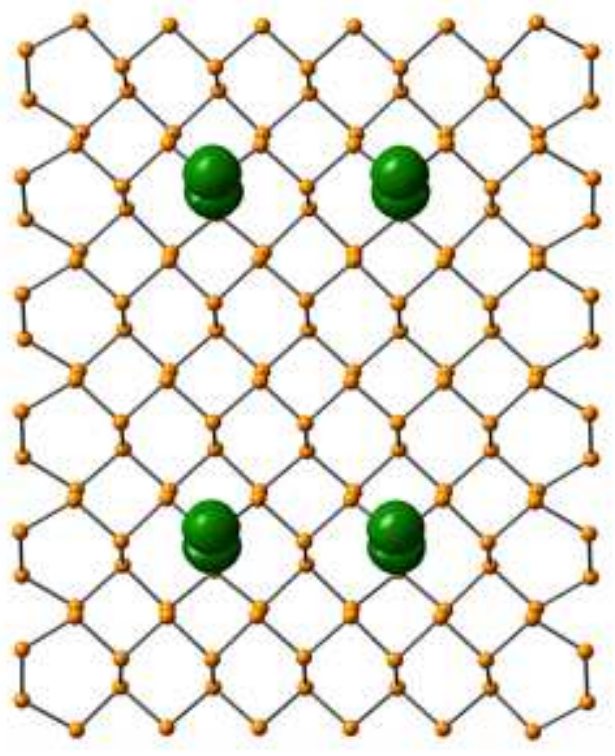

$P(4-S)$

Figure 8

Superposition of single occupied active orbitals for $\mathrm{P}(4-\mathrm{S})$ and $\mathrm{P}(4-\mathrm{Si})$ systems.

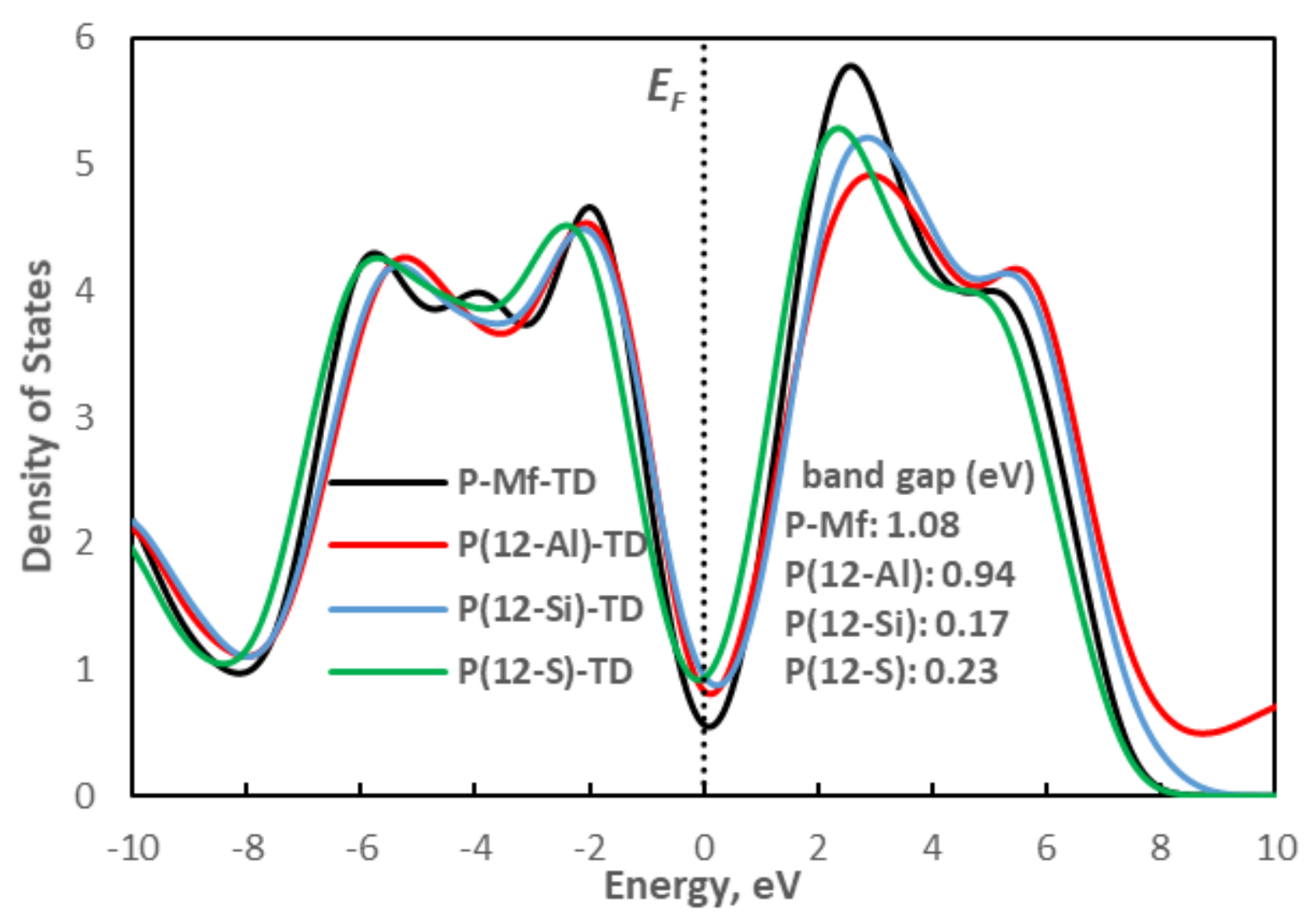

Figure 9 
Total (TD) density of states from TPSS calculations for pristine, P-Mf, and P(12-Al), P(12-Si) and P(12- S). The band gap values are indicated.
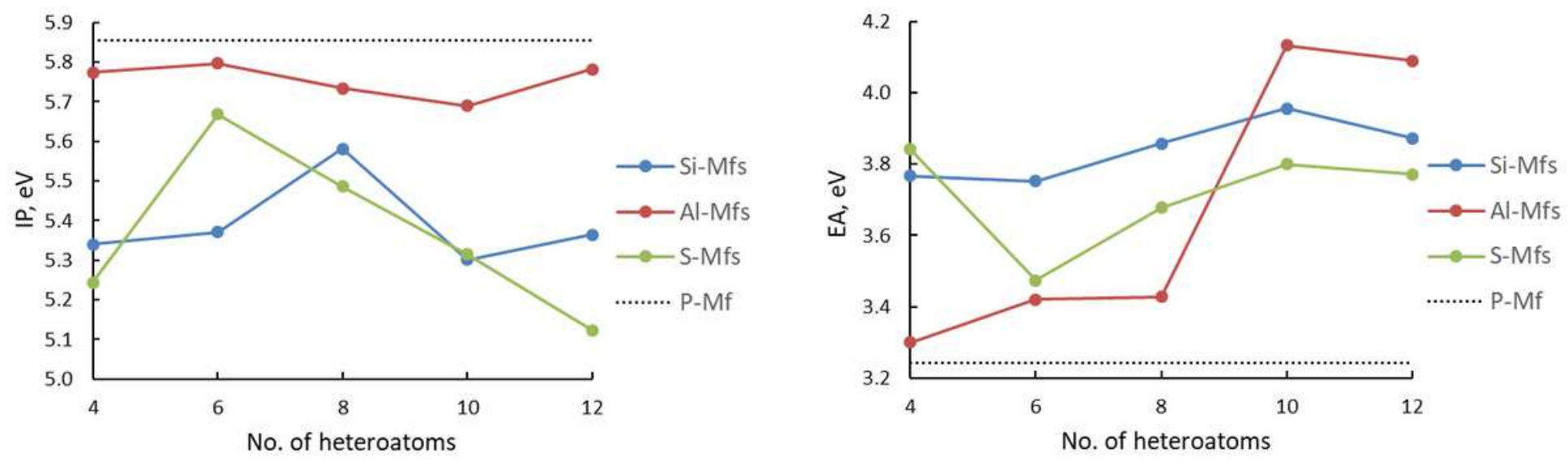

Figure 10

Values of (a) IP and (b) EA, in eV, as a function of the number of heteroatoms in Mfs under study.
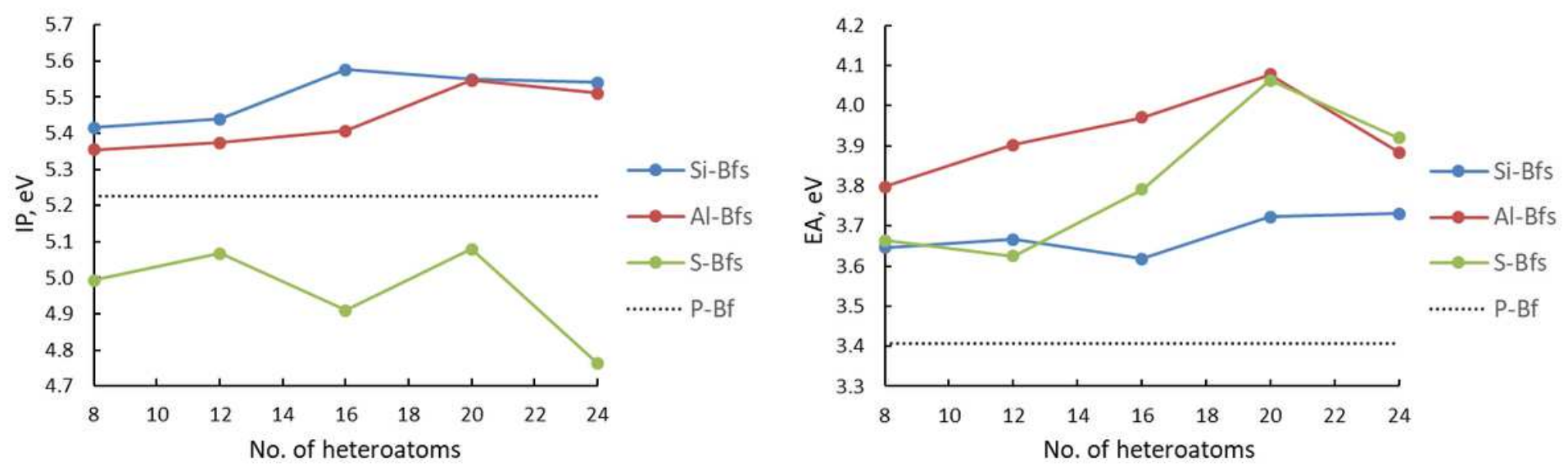

\section{Figure 11}

Values of (a) IP and (b) EA, in eV, as a function of the number of heteroatoms in biflakes under study.
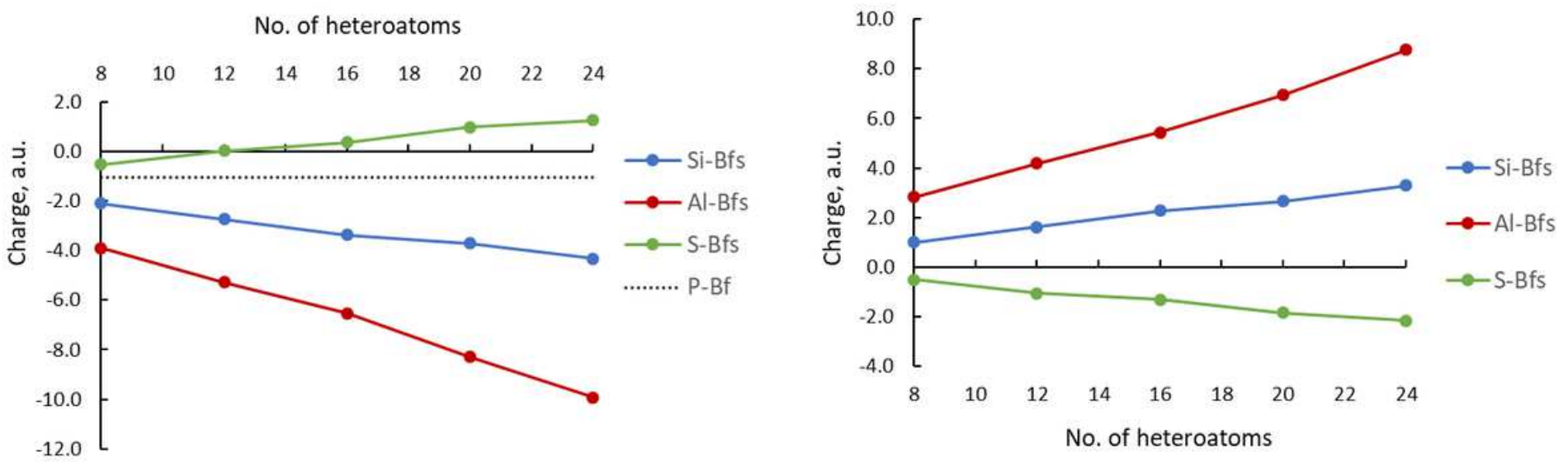
Sum of the NBO charges of (a) the phosphorene skeleton and (b) heteroatoms involved in Bfs.

\section{Supplementary Files}

This is a list of supplementary files associated with this preprint. Click to download.

- Suppinf.pdf 